\title{
Non-Higgsable QCD and the standard model spectrum in F-theory
}

\author{
Antonella Grassi, ${ }^{a}$ James Halverson, ${ }^{b}$ Julius Shaneson ${ }^{a}$ and Washington Taylor ${ }^{c}$ \\ ${ }^{a}$ Department of Mathematics, University of Pennsylvania, \\ 209 S 33rd St, Philadelphia, PA 19104, U.S.A. \\ ${ }^{b}$ Kavli Institute for Theoretical Physics, University of California, \\ 552 University Road, Santa Barbara, CA 93106 U.S.A. \\ ${ }^{c}$ Center for Theoretical Physics, Massachusetts Institute of Technology, \\ 77 Massachusetts Avenue, Cambridge, MA 02139, U.S.A. \\ E-mail: grassi@math.upenn.edu, jim@kitp.ucsb.edu, \\ shaneson@math.upenn.edu, wati@mit.edu
}

ABSTRACT: Many four-dimensional supersymmetric compactifications of F-theory contain gauge groups that cannot be spontaneously broken through geometric deformations. These "non-Higgsable clusters" include realizations of $\mathrm{SU}(3), \mathrm{SU}(2)$, and $\mathrm{SU}(3) \times \mathrm{SU}(2)$, but no $\mathrm{SU}(n)$ gauge groups or factors with $n>3$. We study possible realizations of the standard model in F-theory that utilize non-Higgsable clusters containing SU(3) factors and show that there are three distinct possibilities. In one, fields with the non-abelian gauge charges of the standard model matter fields are localized at a single locus where non-perturbative $\mathrm{SU}(3)$ and $\mathrm{SU}(2)$ seven-branes intersect; cancellation of gauge anomalies implies that the simplest four-dimensional chiral $\mathrm{SU}(3) \times \mathrm{SU}(2) \times \mathrm{U}(1)$ model that may arise in this context exhibits standard model families. We identify specific geometries that realize non-Higgsable $\mathrm{SU}(3)$ and $\mathrm{SU}(3) \times \mathrm{SU}(2)$ sectors. This kind of scenario provides a natural mechanism that could explain the existence of an unbroken QCD sector, or more generally the appearance of light particles and symmetries at low energy scales.

KEYWORDS: F-Theory, Intersecting branes models, Superstring Vacua

ARXIV EPRINT: 1409.8295 


\section{Contents}

1 Introduction $\quad 2$

2 F-theory, non-Higgsable clusters, and seven-branes 5

3 Constructing the standard model spectrum in F-theory 11

3.1 Choose a base $B_{3} \quad 12$

$\begin{array}{ll}3.2 & \text { Check for non-Higgsable clusters } \\ \end{array}$

$\begin{array}{lll}3.3 & \text { Ensure the existence of } \mathrm{SU}(3) \times \mathrm{SU}(2) & 15\end{array}$

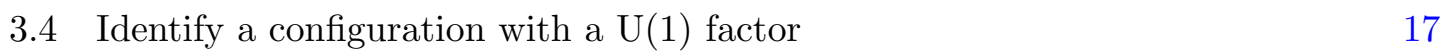

$\begin{array}{ll}3.5 & \text { Low-energy physics, matter, and G-flux } \\ \end{array}$

4 Non-Higgsable QCD $\quad 18$

4.1 Non-perturbative realizations of $\mathrm{SU}(3)$ and $\mathrm{SU}(2) \quad 20$

$4.2 \mathrm{SU}(3) \times \mathrm{SU}(2)$ from a $I V-I I I$ collision 23

5 Examples $\quad \mathbf{3 0}$

5.1 Warm-up: some 6D examples 31

5.1.1 $\mathrm{SU}(2)$ in $6 \mathrm{D}$ models 31

5.1.2 $\mathrm{SU}(3)$ in six dimensions $\quad 32$

5.1.3 $\mathrm{SU}(3) \times \mathrm{SU}(2)$ in six dimensions 33

5.1.4 Non-Higgsable SU(3) in six dimensions 34

$5.24 \mathrm{D}$ models with non-Higgsable $\mathrm{SU}(3)$ groups 35

5.34 D models with non-Higgsable $\mathrm{SU}(3) \times \mathrm{SU}(2) \quad 38$

6 Directions for further development $\quad 39$

$\begin{array}{lll}6.1 \text { Abelian factors } & 39\end{array}$

6.2 The the low-energy physical theory and G-flux 42

$\begin{array}{lll}7 & \text { Summary and discussion } & \mathbf{4 5}\end{array}$

7.1 A summary of non-Higgsable QCD 45

7.2 The minimal chiral $\mathrm{SU}(3) \times \mathrm{SU}(2) \times \mathrm{U}(1)$ model gives standard model generations 46

$\begin{array}{lll}7.3 & \text { Discussion } & 47\end{array}$ 


\section{Introduction}

In recent years there has been much progress in understanding the physics of F-theory [1-3] compactifications. An F-theory compactification to $d=4,6$ or 8 space-time dimensions can be thought of as a supersymmetric type IIB compactification with a varying axiodilation profile $\tau=C_{0}+i / g_{s}$; another definition that is more precise for some purposes arises by considering an M-theory compactification to $d=3,5$, or 7 dimensions on an elliptically fibered Calabi-Yau manifold in a vanishing fiber limit that corresponds to a decompactification limit via a single T-duality. The set of F-theory compactifications represents a promising region of the landscape for a number of reasons. One is that grand unified models in F-theory have some features that are more realistic than their weakly coupled type II counterparts - though as we show in this paper, even in models without grand unification F-theory has certain desirable phenomenological features not present in the weakly coupled limit. Perhaps most importantly, F-theory appears to provide the broadest view currently available of the landscape of $\mathcal{N}=1$ string compactifications to six or four dimensions. See $[4-6]$ and $[7,8]$ respectively for pedagogical and phenomenological reviews of F-theory.

An interesting feature of many string compactifications is the presence of "nonHiggsable clusters" [9]. These are connected gauge sectors, carried by seven-branes in F-theory, that cannot be spontaneously broken. The simplest non-Higgsable clusters consist of a single gauge group with little or no charged matter. It has been known since the early days of F-theory that certain gauge groups, particularly $\mathrm{SU}(3), \mathrm{SO}(8), F_{4}, E_{6}, E_{7}$, and $E_{8}$, can be realized through dual heterotic and F-theory compactifications in models with no charged matter, so that these groups cannot be Higgsed [3]. Non-Higgsable clusters can also contain multiple gauge group factors and charged matter. A systematic classification of these clusters for six-dimensional F-theory models was given in [9], and includes non-Higgsable gauge group products such as $G_{2} \times \mathrm{SU}(2)$ with jointly charged matter.

Such non-Higgsable clusters with multiple gauge group factors and matter are unique to F-theory in known string constructions. Mathematically, non-Higgsable clusters are produced when the elliptically fibered Calabi-Yau manifold $X$ used for an F-theory compactification has certain types of singularities at generic points in its complex structure moduli space $C S(X)$. Physically, from the point of view of the corresponding low-energy theory, a non-Higgsable structure arises when there is no symmetry-breaking flat direction in the supersymmetric moduli space. For six-dimensional theories, there is a direct correspondence between the geometric structure and the non-Higgsability of the low-energy theory.

We emphasize at the outset that the situation is more complicated in four-dimensional theories, since there are additional features that affect the low-energy physics. In particular, though a four-dimensional F-theory compactification with a geometrically non-Higgsable cluster exhibits seven-brane gauge sectors that cannot be broken by complex structure deformation, we cannot rule out the possibility of other effects that may, in certain cases, break the gauge group. These include additional moduli and a set of discrete fluxes; the latter corresponds to $G_{4}$ flux in the M-theory picture, which we henceforth refer to as G-flux in the F-theory picture. There are often many discrete flux choices (in fact for some $X$ the G-flux must always be non-trivial), and depending on the choice there are 
at least three possible effects on the low-energy theory: it may induce a flux-breaking of the gauge group, give rise to a chiral spectrum, and/or stabilize some of the complex structure moduli of $X$. Though we focus primarily on the geometry of $X$ and its complex structure, the scenario we propose operates under the assumptions that one does not turn on fluxes that break the geometrically imposed gauge group $G$ (as typically assumed in the F-theory GUT literature) or force an enhancement of $G$ through moduli stabilization on a locus with non-generic gauge group. We discuss these issues in section 6.2 , where we also present a discussion of the relationship between geometric and physical non-Higgsability in four-dimensional theories. Throughout this paper we identify and study geometrically non-Higgsable clusters, which correspond to seven-brane sectors that cannot be broken by complex structure deformation; in principle we expect that these will generally correspond to non-Higgsable structure in the resulting low-energy 4D supergravity theory, but in some cases this relationship may be subject to the caveats above.

The complete set of gauge group factors that can be associated to a non-Higgsable geometry is rather restricted. In decreasing order of dimension, the possible simple (or abelian) factors $G_{i}$ in a non-Higgsable gauge group $G=\prod_{i} G_{i}$ (in any dimension) are in the set: ${ }^{1}$

$$
\left\{E_{8}, E_{7}, E_{6}, F_{4}, \mathrm{SO}(8), \mathrm{SO}(7), G_{2}, \mathrm{SU}(3), \mathrm{SU}(2), \mathrm{U}(1)\right\} \text {. }
$$

Notably, SU(5) and SO(10) are both absent from this list and SU(3) and SU(2) are the only possible $\mathrm{SU}(n)$ groups. The singularities that may give rise to a non-Higgsable $\mathrm{SU}(3)$ or $\mathrm{SU}(2)$ (or any other non-Higgsable gauge group factor) do not admit a description in terms of perturbative string theory on $D 7$ branes; they arise from non-trivial $(p, q)$ seven-branes.

Though in six dimensions the only non-Higgsable product groups that can arise are $G_{2} \times \mathrm{SU}(2)$ and $\mathrm{SU}(2) \times \mathrm{SO}(7) \times \mathrm{SU}(2)$ (again, up to possible quotients by a discrete subgroup), in four dimensions the set of possibilities is richer. As we show in this paper, this includes the possibility of an $\mathrm{SU}(3) \times \mathrm{SU}(2)$ non-Higgsable cluster or a non-Higgsable $\mathrm{SU}(3)$ with Higgsable $\mathrm{SU}(2)$. In both cases, we will see that the specific spectrum of charged matter exhibited by the geometry is relevant for particle physics. Regarding the first of these possibilities, while of course the $\mathrm{SU}(2)$ of the standard model is in fact broken in the infrared by the Higgs field itself, in some reasonable scenarios this occurs through radiative electroweak symmetry breaking and may still be compatible with a non-Higgsable SU(2) in the model with unbroken SUSY.

It is worth emphasizing that the same local singularity types that can give nonHiggsable seven-branes carrying specific gauge group factors can also arise in other geometries from Higgsable seven-brane configurations carrying the same gauge group factors but with (in general) more charged matter; in contrast, there are other singularities, and associated gauge group factors, that may never be non-Higgsable. From the F-theory point of view, many different gauge groups with a rich variety of possible matter spectra can be arranged by tuning the axiodilaton profile $\tau$ over a given compactification space; this corre-

\footnotetext{
${ }^{1}$ Note that the actual gauge group can also involve a quotient by a discrete group, so that strictly speaking one should say that the set above gives the complete list of possible gauge algebras. In this paper we do not worry about the global structure of the group, and simply refer to the various simple group factors.
} 
sponds geometrically to tuning the structure of the elliptically fibered Calabi-Yau manifold by varying the parameters in a Weierstrass model, as described in more detail in the next section. Such tuning, which can give rise to (Higgsable versions of) the gauge group types mentioned above as well as many other types including general $\mathrm{SU}(n)$ and $\mathrm{SO}(n)$ factors, has been the primary approach taken to F-theory model building to date.

In this paper we give a systematic description of the different possible ways that the nonabelian $\mathrm{SU}(3)$ and $\mathrm{SU}(2)$ factors in the standard model can be realized using geometrically non-Higgsable and/or Higgsable structures. We focus in particular on possible F-theory realizations of the standard model that utilize a non-Higgsable SU(3) factor; there are three qualitatively different possibilities in this case for how the $\mathrm{SU}(2)$ factor is realized.

Constructions with such a non-Higgsable QCD sector yield two important physical features. The first is that the type $I V$ singular fiber that realizes a non-Higgsable $\mathrm{SU}(3)$ factor gives rise to a rich matter spectrum when it intersects singularity carrying an $\mathrm{SU}(2)$ gauge group; intuitively, this is because the type $I V$ fiber (without monodromy) realizes an $\mathrm{SU}(3)$ gauge theory on four $(p, q)$ seven-branes, as opposed to an $\mathrm{SU}(3)$ gauge factor produced from three D7-branes at weak coupling. We find in particular that fields carrying all of the representations of $\mathrm{SU}(3) \times \mathrm{SU}(2)$ needed for the complete set of standard model matter matter fields may be localized at the $\mathrm{SU}(3) \times \mathrm{SU}(2)$ intersection obtained by the intersection of a type IV and type III fiber; this is in sharp contrast to the type IIB $\mathrm{SU}(3) \times \mathrm{SU}(2)$ intersection of two stacks of D7-branes, which realizes only bifundamental matter that has the quantum numbers of quark doublets.

The second potentially interesting feature of a non-Higgsable $\mathrm{SU}(3)$ factor is that it provides a natural mechanism that could explain the existence of an unbroken QCD sector in nature. While this is not an issue in the standard model itself, the necessary existence of electromagnetically charged and/or colored scalars in supersymmetric theories allows for the possible existence of charge or color-breaking (CCB) vacua in supersymmetric extensions of the standard model. In the MSSM the absence of dangerous CCB vacua places bounds on soft supersymmetry breaking terms, which can rule out or significantly constrain specific models; this issue is discussed further in section 7 .

More broadly, the constructions we describe here may have some relevance for the question of why nature provides us with symmetries and light particles at low energy scales. In both field theory and string theory, mechanisms have been proposed for moduli stabilization at enhanced symmetry points; see e.g. [10] and references therein. Such mechanisms are motivated in part by the presence of an unbroken QCD sector with a confinement scale far below the Planck mass and the common expectation that typical vacua in the landscape break gauge symmetry at high scale. In much of the literature in this area, in fact, enhanced symmetry points are assumed to be relatively rare and in particular non-generic in the supersymmetric moduli space. If this assumption is true, then indeed some mechanism would be desirable to explain why vacua are stabilized at those special points in the moduli space that have enhanced symmetries; the authors of [10] and other related works also seek to develop mechanisms that explain the cosmological dynamics that might drive the theory to these vacua. 
Notably, F-theory compactifications with non-Higgsable clusters sidestep much of this issue, since they exhibit gauge symmetry at generic points in their supersymmetric moduli space. In particular, in the kind of QCD scenarios that we describe here, the existence of an unbroken QCD sector does not require stabilization at a special locus in moduli space: a generic point will suffice. Though conventional SU(5) grand unification is impossible in this scenario, it is particularly intriguing that the theory singles out $\mathrm{SU}(3)$ and $\mathrm{SU}(2)$ from all other $\mathrm{SU}(n)$ and leads naturally toward the spectrum of the standard model.

This paper is organized as follows. In section 2 we review some basics of F-theory and non-Higgsable clusters. Section 3 gives a systematic description of the possible ways that any F-theory model may be constructed that contains within it the $\mathrm{SU}(3) \times \mathrm{SU}(2) \times \mathrm{U}(1)$ of the standard model gauge group. In section 4 we introduce non-Higgsable QCD and its three qualitatively different realizations. Utilizing a string junction analysis, we study the seven-branes associated with Kodaira type $I V$ and $I I I$ singularities, and also the structure of a $I V-I I I$ intersection, which turns out to generically involve an extra brane carrying an $I_{1}$ singular fiber. Section 5 contains a variety of examples of specific F-theory compactifications to $6 \mathrm{D}$ and $4 \mathrm{D}$ that illustrate the general ideas of the paper and serve as existence proofs for the various types of non-Higgsable SU(3) models; these examples could be analyzed in more detail in future work. In section 6 we discuss some of the technical issues that must be addressed to describe the detailed structure of low-energy supergravity theories that incorporate a geometrically non-Higgsable $\mathrm{SU}(3)$.

The reader who is interested in our results, but may not be as familiar with F-theory, may find it useful to read the final section (section 7) first. In this concluding section, we summarize our results on the three qualitatively different possible realizations of nonHiggsable QCD and show that the cancellation of gauge anomalies implies that the simplest four-dimensional chiral $\mathrm{SU}(3) \times \mathrm{SU}(2) \times \mathrm{U}(1)$ model arising from a non-Higgsable QCD scenario exhibits standard model families. We also discuss the physical implications of this scenario, in particular with regard to model building and enhanced symmetry points in the string landscape.

\section{F-theory, non-Higgsable clusters, and seven-branes}

Let us briefly review some relevant facts about F-theory, $(p, q)$ seven-branes, and nonHiggsable clusters.

An F-theory compactification is most easily thought of as a generalization of type IIB string theory. Although the M-theory description is more complete for some purposes, we will focus on the physics of seven-branes, which are determined geometrically by promoting the type IIB axiodilaton $\tau=C_{0}+i / g_{s}$ to be the complex structure modulus of an elliptic curve (i.e., a complex torus with a marked point). A supersymmetric F-theory compactification is defined by compactifying type IIB on a complex manifold $B$ with an axiodilaton profile that defines an elliptically fibered Calabi-Yau manifold $X$, where a projection $\pi: X \rightarrow B$ gives the fibration, with $\pi^{-1}(p)=\mathbb{E}$ an elliptic curve at each point on $B$. Though the Kähler modulus of the elliptic fiber vanishes in the F-theory limit, its complex structure remains intact and determines the structure of seven-branes. The 
elliptic fiber can become degenerate along codimension one loci that mark the presence of type IIB seven-branes, and the axiodilaton undergoes a nontrivial $\mathrm{SL}(2, \mathbb{Z})$ monodromy along a curve that goes around such a seven-brane. For us it will be critical that F-theory contains a broader set of possible seven-branes than the D7-branes and O7-planes of the weakly coupled type IIB theory; for example, seven-brane configurations can arise that carry exceptional gauge groups and non-perturbative realizations of $\mathrm{SU}(3)$ and $\mathrm{SU}(2)$.

Seven-branes associated with localized singularities in the function $\tau$ on $B$ determine many aspects of the gauge sectors in an F-theory compactification; others can arise from D3branes, which we do not consider here. The structure of seven-branes is determined as follows. The elliptically fibered Calabi-Yau variety $X$ can be described in Weierstrass form [11]

$$
y^{2}=x^{3}+f x+g,
$$

where $f$ and $g$ are functions that depend on coordinates in $B$. Specifically, $f$ and $g$ are sections of the line bundles $\mathcal{O}\left(-4 K_{B}\right)$ and $\mathcal{O}\left(-6 K_{B}\right)$, where $K_{B}$ is the canonical bundle of $B$. Seven-branes are located along the discriminant locus $\Delta=0$, where

$$
\Delta=4 f^{3}+27 g^{2}
$$

This is a complex codimension one locus in $B$ along which the elliptic fiber becomes singular. The possible singular fibers in codimension one (originally analyzed for elliptic surfaces) have been classified by Kodaira [12, 13].

Up to an effect known as outer monodromy, which we will discuss later, the gauge group is determined by the singular fibers over the irreducible complex codimension one loci (divisors) $D$ that comprise the discriminant locus; the individual gauge group factors can be identified easily from the structure of $f, g$, and $\Delta$. Specifically, if $f, g, \Delta$ vanish to specific orders on $D$,

$$
f \sim z^{\operatorname{ord}(f)} \quad g \sim z^{\operatorname{ord}(g)} \quad \Delta \sim z^{\operatorname{ord}(\Delta)}
$$

where $z$ is a local complex coordinate on $B$ with $D=\{z=0\}$, then the type of singular fiber above a generic point in $D$ can be read off from table 1 ; a seven-brane is located along $z=0$. In this paper we often use explicit coordinates such as $z$, which can be thought of as local coordinates on a general complex manifold, or homogeneous (Cox) coordinates when the manifold has a toric description. Divisors on which $f, g$ vanish to orders $(4,6)$ are associated with non-minimal singularities that cannot be resolved to a Calabi-Yau total space. Such singular geometries lie at an infinite distance from any fixed smooth geometry in the complex structure moduli space, and we do not consider them here. Codimension two loci where $(f, g)$ vanish to degrees $(4,6)$ correspond to branches in the moduli space associated with tensionless string transitions [3, 14]; these singular Weierstrass models can be interpreted locally in terms of a superconformal field theory ([15] see e.g. [16-19] for recent work); a smooth Calabi-Yau resolution does not exist unless one first performs a blow-up of the codimension two locus in the base. In this paper we do not consider geometries with codimension two $(4,6)$ loci. 


\begin{tabular}{|ccccc|}
\hline Fiber Type & ord $(\mathrm{f})$ & ord $(\mathrm{g})$ & ord $(\Delta)$ & Gauge Group \\
\hline smooth & $\geq 0$ & $\geq 0$ & 0 & - \\
$I_{n}$ & 0 & 0 & $n$ & $\mathrm{SU}(n)$ \\
$I I$ & $\geq 1$ & 1 & 2 & - \\
$I I I$ & 1 & $\geq 2$ & 3 & $\mathrm{SU}(2)$ \\
$I V$ & $\geq 2$ & 2 & 4 & $\mathrm{SU}(3)$ \\
$I_{n}^{*}$ & 2 & $\geq 3$ & $n+6$ & $\mathrm{SO}(2 n+4)$ \\
$I_{n}^{*}$ & $\geq 2$ & 3 & $n+6$ & $\mathrm{SO}(2 n+4)$ \\
$I V^{*}$ & $\geq 3$ & 4 & 8 & $E_{6}$ \\
$I I I^{*}$ & 3 & $\geq 5$ & 9 & $E_{7}$ \\
$I I^{*}$ & $\geq 4$ & 5 & 10 & $E_{8}$ \\
\hline
\end{tabular}

Table 1. Kodaira's classification of singular fibers, together with the associated gauge group (up to outer monodromy, which affects compactifications to $6 \mathrm{D}$ and $4 \mathrm{D}$ ) in F-theory and degrees of vanishing along $f, g$, and $\Delta$. Note that $\mathrm{SU}(3)$ and $\mathrm{SU}(2)$ are on a special footing compared to the other $\mathrm{SU}(n)$ groups.

Note from table 1 that a distinguished role is played by $\mathrm{SU}(3)$ and $\mathrm{SU}(2)$; unlike $\mathrm{SU}(n>3)$, which can only be realized by an $I_{n}$ fiber, $\mathrm{SU}(3)$ and $\mathrm{SU}(2)$ may also be realized through a type $I V$ or type $I I I$ fiber. One fundamental difference between these singularity types is that the type $I V$ and $I I I$ singularity types are automatically imposed when $f, g$ vanish to specified orders (as is also the case for the $I_{0}^{*}, I V^{*}, I I I^{*}$ and $I I^{*}$ types of singular fibers), while the type $I_{n}$ singularities (like the type $I_{n>0}^{*}$ singularities) require the cancellation of nonzero terms in $f, g$ for a higher degree of cancellation in $\Delta$. This difference underlies the distinction between those gauge groups that can be realized in non-Higgsable clusters, and those that cannot.

A non-Higgsable cluster arises when F-theory on $X$ exhibits a gauge sector that cannot be spontaneously broken by a complex structure deformation; that is, when the singularities associated to the seven-branes exist at a generic point in the complex structure moduli space of $X, C S(X)$. Mathematically, this occurs when a connected set of complex codimension one loci (divisors) $D_{i}$ are irreducible components of $f$ and $g$; that is, $f$ and $g$ always vanish on $D_{i}$. When $\operatorname{ord}_{D_{i}}(f) \geq 1, \operatorname{ord}_{D_{i}}(g) \geq 2$, this forces a Kodaira singularity in $X$ associated with a non-trivial gauge group. This gauge group is, in fact, the gauge group of an associated six-dimensional F-theory compactification for a generic point in $C S(X)$; for this to be true in four dimensions, it is also necessary to choose G-flux such that it does not affect the gauge group.

The presence of non-Higgsable gauge groups can be seen easily in many examples; if for the most generic $f$ and $g$ for a given base $B$ the discriminant satisfies $\Delta \sim z^{n}$ for some $n>0$ and a local coordinate $z$, then there is a seven-brane along $z=0$, and if $n>2$ then the seven-brane configuration carries a non-Higgsable gauge group. Alternatively, if $\Delta \sim z^{n}$ with $n>2$ only for a specialization in the complex structure of $X$, i.e., for non- 
generic $f$ and $g$, then moving to a generic point in moduli space describes spontaneous symmetry breaking via the Higgs mechanism. Such a symmetry breaking mechanism does not exist for non-Higgsable clusters.

It is straightforward to determine which individual gauge group factors can possibly arise in a non-Higgsable cluster. As discussed above, these are precisely the gauge group factors associated with Kodaira singularity types where the degree of vanishing of $\Delta$ is forced by the degrees of vanishing of $f, g$. This follows since in general $f, g$ can be described as linear combinations of monomials with free complex coefficients, and the only way a cancellation can be arranged in $\Delta$ to a higher order than $\min (3 \operatorname{ord}(f), 2 \operatorname{ord}(g))$ is by choosing non-generic values of the coefficients in $f, g$. Thus, the gauge group factors that can arise from a non-Higgsable structure are those associated with all Kodaira singularity types except $I_{n}$ and $I_{n>0}^{*}$.

For example, consider the question of whether a type $I V$ fiber may be non-Higgsable; assume that there exists a base $B$ and a local coordinate $z$ such that for the most general $f$ and $g$ we have

$$
f=z^{2} \tilde{f} \quad \text { and } \quad g=z^{2} \tilde{g}
$$

i.e., one can factor out an overall $z^{2}$ out of both $f$ and $g$. If this is the case, we see that $\Delta=z^{4}\left(4 z^{2} \tilde{f}^{3}+27 \tilde{g}^{2}\right)$, and note that $\Delta$ automatically has the correct order of vanishing for a type $I V$ fiber; i.e., without requiring any specialization in moduli space. This shows that a type $I V$ fiber may in principle arise as a non-Higgsable cluster. Alternatively, note that if one engineers a seven-brane with $\mathrm{SU}(n)$ gauge symmetry arising from an $I_{n}$ fiber, the order of vanishing of $f$ and $g$ along $z=0(\operatorname{ord}(f)=\operatorname{ord}(g)=0)$ does not ensure $\operatorname{ord}(\Delta)=n$; an additional tuning is always needed, and thus an $I_{n}$ fiber cannot give rise to a non-Higgsable cluster.

This simple analysis shows that fibers of type $I V^{*}, I I I^{*}, I I^{*}, I V, I I I, I I$, and $I_{0}^{*}$ may be realized as factors in a non-Higgsable cluster. There are no non-Higgsable clusters in eight dimensions, since in eight dimensions all F-theory vacua are part of a single moduli space where all nonabelian gauge group factors can be Higgsed. To determine the complete set of gauge group factors that can be associated with the non-Higgsable Kodaira singularity types for F-theory compactifications to $6 \mathrm{D}$ or $4 \mathrm{D}$, we must consider additional structure known as outer monodromy that can act on a fiber in such a way that the simply-laced gauge group factor becomes non-simply laced [20]. This occurs when a closed path on a divisor $D$ carrying a nontrivial Kodaira singularity type brings the resolved fiber back to itself up to a nontrivial permutation on its topological structure, which as Kodaira showed is encoded by a set of rational curves $\left(\mathbb{P}^{1}\right.$ 's) connected in the structure of the associated Dynkin diagram. For example, there is an action of $S_{3}$ on the Dynkin diagram of $\mathrm{SO}(8)$; from a $\mathbb{Z}_{3}$ subgroup of this action we see $\mathrm{SO}(8)$ become $G_{2}$ as

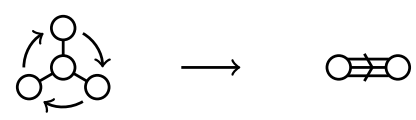

where the nodes mapped to one another under the action are identified. A geometry exhibiting this behavior gives rise to a $G_{2}$ gauge theory. Similarly, outer monodromy can 


\begin{tabular}{|ccc|}
\hline Fiber Type & Outer Monodromy & Gauge Group \\
\hline$I I^{*}$ & - & $E_{8}$ \\
$I I I^{*}$ & - & $E_{7}$ \\
$I V^{*}$ & - & $E_{6}$ \\
$I V^{*}$ & $\mathbb{Z}_{2}$ & $F_{4}$ \\
$I_{0}^{*}$ & - & $\mathrm{SO}(8)$ \\
$I_{0}^{*}$ & $\mathbb{Z}_{2}$ & $\mathrm{SO}(7)$ \\
$I_{0}^{*}$ & $S_{3}$ & $G_{2}$ \\
$I V$ & - & $\mathrm{SU}(3)$ \\
$I V$ & $\mathbb{Z}_{2}$ & $\mathrm{Sp}(1) \cong \mathrm{SU}(2)$ \\
$I I I$ & - & $\mathrm{SU}(2)$ \\
$I I$ & - & - \\
\hline
\end{tabular}

Table 2. Singular fibers in Kodaira's classification that may give rise to non-Higgsable clusters, together with their possible outer monodromies and associated gauge groups.

reduce the $\mathrm{SU}(3)$ gauge group on a type $I V$ singularity to $\mathrm{Sp}(1)$ (which has the same Lie algebra as $\mathrm{SU}(2))$.

Taking outer monodromy into account, the possible gauge groups that can appear on a non-Higgsable seven-brane are given in table 2. In each case, the existence and type of outer monodromy can be determined by the structure of the Weierstrass coefficients $f, g[20,21]$. In the case of interest to us here, for a type $I V$ singularity the gauge group is $\mathrm{SU}(2)$ unless the leading coefficient in the expansion of $g$ in a local coordinate $z$ around the locus in the base supporting the singularity is a perfect square, i.e., $g=g_{2} z^{2}+g_{3} z^{3}+\cdots=$ $z^{2} \gamma^{2}+\mathcal{O}\left(z^{3}\right)$, where $\gamma$ can be an algebraic function of the remaining coordinates. For a type $I V$ singularity in a non-Higgsable cluster to give rise to a non-Higgsable $\mathrm{SU}(3)$ gauge group, the coefficient $g_{2}$ must be a single (even) monomial or a constant, or it is not generically a perfect square. We will present examples of this type.

While the preceding discussion describes individual gauge factors in a non-Higgsable cluster, it is also possible for non-Higgsable clusters to contain multiple gauge group factors as well as matter charged under these gauge group factors. For six dimensions, the set of possible non-Higgsable clusters of gauge groups and associated minimal matter content for F-theory compactifications on Calabi-Yau threefolds over base surfaces $B_{2}$ were classified in [9]. As mentioned above, these include clusters that give rise to $G_{2} \times \mathrm{SU}(2)$ and $\mathrm{SU}(2) \times$ $\mathrm{SO}(7) \times \mathrm{SU}(2)$ gauge groups, with matter that is jointly charged under each adjacent pair of gauge groups in the cluster. A similar set of clusters are present at the geometric level for base threefolds. For 4D F-theory compactifications with smooth heterotic duals, the only possible non-Higgsable clusters are those with only a single gauge group factor (but include $\mathrm{SU}(2)$, which cannot be realized by itself as a non-Higgsable gauge group in $6 \mathrm{D}$ compactifications); an analysis of the non-Higgsable structures in F-theory models with 
smooth heterotic duals was carried out in [22]. The range of product groups that can be realized in non-Higgsable clusters also increases substantially for four dimensional F-theory compactifications [23]. In particular, as we demonstrate with some specific examples in this paper, it is possible in a $4 \mathrm{D}$ F-theory compactification to have a non-Higgsable cluster with the gauge group structure $\mathrm{SU}(3) \times \mathrm{SU}(2)$.

The discussion so far has focused on the gauge group of an F-theory compactification. Of course, for a complete specification of the physics of a low-energy theory arising from F-theory we also need to describe the matter spectrum of charged particle states that arise in the theory, and the associated representation theory.

A geometric description of the physics of general seven-brane configurations that gives substantial insight into the nonperturbative structure of these objects is given using the particle states that end on seven-branes in F-theory. These states can be described using the language of $(p, q)$ string junctions [24-26]. $(p, q)$ strings are bound states of $p$ F-strings and $q$ D-strings; these are the F-theory limit of particular M2-brane configurations in a defining M-theory compactification, namely those where the M2-brane wraps the one-cycle $p \pi_{1}+$ $q \pi_{2}$ in the elliptic fiber, as well as extending in $B$. Upon movement in the complex structure moduli space of $X$, these strings, and more complicated branched string junctions formed from them, can shrink to zero size, in which case they become massless. If this occurs along a codimension one locus in $B$, the string junctions describe vector multiplets; if along a codimension two locus, they live in matter multiplets (i.e., hypermultiplets and chiral/antichiral multiplets in six- and four-dimensional compactifications, respectively). For recent studies of the connection between deformation theory and string junctions, see [27, 28].

The representation theoretic matter content can also be read off by performing a Kähler resolution in M-theory [20, 29]. This type of resolution of singularities has been used to analyze the representation theory structure of matter in a wide variety of geometries. Despite a substantial amount of work on this topic (e.g. [30-36]), there is still no complete classification of the types of codimension two singularities that can arise in F-theory, and no complete dictionary that relates such codimension two singularities to arbitrary representations of the possible gauge groups. For the purposes of this paper, however, we will only need a simple set of representations, namely matter states that transform in the fundamental representation of a single $\mathrm{SU}(n)$ gauge factor, or bifundamental representations that transform in the fundamental (or anti-fundamental) of each of a pair of product gauge factors $\mathrm{SU}(n) \times \mathrm{SU}(m)$.

One important point that we emphasize again here is that while in six dimensions the gauge group and matter content encoded in the geometry of an F-theory compactification correspond precisely to the physical gauge group and matter content, in four dimensions the connection is much less direct. Additional features such G-flux, D3-branes, and additional moduli are present in 4D F-theory compactifications, and can modify both the gauge group and matter content of the physical theory from those which are seen in the geometric description in terms of the Calabi-Yau fourfold. In particular, a detailed determination of the matter content of a $4 \mathrm{D}$ F-theory compactification depends upon consideration of these factors. In this paper we primarily analyze the geometric structure of compactifications, and assume that in a broad class of cases the physical structure of the low-energy theory 
will match the geometric structure, as often assumed in the F-theory GUT literature. We address some of the issues involved in connecting the geometric picture to the low-energy physics and determining precise features of the matter spectrum in sections 6 and 7 .

\section{Constructing the standard model spectrum in F-theory}

In this section we describe in very general terms the structure of any $4 \mathrm{D}$ F-theory model that contains the gauge group $G_{321}=\mathrm{SU}(3) \times \mathrm{SU}(2) \times \mathrm{U}(1)$ at the level of geometry; in particular, we allow for the use of non-Higgsable clusters. The basic approach taken is based on the geometry of the base $B_{3}$ of the elliptically fibered Calabi-Yau fourfold $X, \pi: X \rightarrow B_{3}, \pi^{-1}(p) \cong \mathbb{E} \forall p \in B_{3} \backslash \Delta$. By focusing on the structure of the base $B_{3}$ many aspects of the structure and classification of possible models are simplified. In particular, for any given base $B_{3}$ there can be many different elliptically fibered Calabi-Yau varieties with different singularity structures associated with different gauge groups, realized by tuning to substrata in complex structure moduli, i.e. to a specialized Weierstrass model. By grouping these together and focusing on the physics of the generic elliptic fibration over any given base, the classification problem is substantially simplified.

In broad strokes, any $4 \mathrm{D}$ F-theory model that contains the gauge group $G_{321}$ can be constructed and described in the following steps:

A) Choose a base $\boldsymbol{B}_{\mathbf{3}}$. A threefold must be chosen that can support an elliptically fibered fourfold. Many examples of such threefolds are known, though the complete set of possibilities has not been classified.

B) Check for non-Higgsable clusters. From the most general Weierstrass model over a given base $B_{3}$ it is possible to determine whether or not the geometry exhibits a non-Higgsable cluster. In particular, we can check to see if there are $\mathrm{SU}(3)$ and/or $\mathrm{SU}(2)$ non-Higgsable clusters. We utilize several different methods in this paper to check for the existence of non-Higgsable clusters in 4D F-theory compactifications.

C) Tune non-abelian factors as necessary. If the non-Higgsable part of the gauge group does not contain $\mathrm{SU}(3) \times \mathrm{SU}(2)$, the rest of the nonabelian part of $G_{321}$ can be tuned by going to a special locus ${ }^{2}$ in the moduli parameterizing the generic Weierstrass model over $B_{3}$.

D) Identify a configuration with a $\mathbf{U}(1)$ factor. F-theory models with abelian $\mathrm{U}(1)$ factors correspond to Calabi-Yau manifolds with nontrivial rational sections that live in a Mordell-Weil group of nonzero rank. Identifying the Mordell-Weil group of a Weierstrass model over a given base is generally a difficult mathematical problem, but methods exist for constructing general models with a single U(1) factor over any given base.

\footnotetext{
${ }^{2}$ Note that this may not be possible in some bases. For example, there are bases $B_{3}$ that contain a non-Higgsable SU(3), where there is no possible tuning of a Weierstrass model having an additional SU(2) on an intersecting divisor without producing a $(4,6)$ singularity at a codimension two locus on the base.
} 
Of course, obtaining $G_{321}$ is necessary but not sufficient to realize a model that contains the standard model spectrum; the geometry must exhibit particular representations of this group in order to match the field content of the SM. In the MSSM, for example, the matter fields are chiral superfields in representations of $G_{321}$

$$
\begin{array}{lll}
Q:(3,2)_{1} & U:(\overline{3}, 1)_{-4} & D:(\overline{3}, 1)_{2} \\
L:(1,2)_{-3} & E:(1,1)_{6} & N:(1,1)_{0} \\
H_{u}:(1,2)_{3} & H_{d}:(1,2)_{-3} &
\end{array}
$$

and obtaining a realistic theory requires identifying the fields $Q, U, D, L, E$, at the very least, possibly augmented by $N, H_{u}$, and $H_{d}$ and/or other representations depending on the model. Identification of the representations of $G_{321}$ that appear in the geometry can be done following step (D), by analyzing codimension two singularities using deformation or resolution methods. As discussed earlier, we focus here purely on the geometric analysis of the gauge group and matter content. A more complete analysis would need to incorporate G-flux and other features that might affect the low-energy matter spectrum; in particular, though the correct Lie algebra representations of the standard model may emerge geometrically, obtaining a chiral spectrum requires the introduction of G-flux.

For models with a suitable spectrum, in principle more detailed aspects of the standard model could be checked. In this paper we primarily focus on the construction of models that have the nonabelian $\mathrm{SU}(3) \times \mathrm{SU}(2)$ structure of the standard model, though we also carry out a limited analysis of $\mathrm{U}(1)$ factors and matter spectra both in general and in specific cases.

In principle, this approach could be used to systematically identify large classes of F-theory models that contain the gauge group $G_{321}$ that appears in the standard model of particle physics. More generally, this approach could be used to systematically describe elliptically fibered Calabi-Yau manifolds that give F-theory models with any gauge group. A more detailed description of how this general approach can in principle be used to describe all elliptically fibered Calabi-Yau threefolds, and some technical challenges to a complete classification using this approach are described in [37]. In addition to the issues described in that paper, for fourfolds there is a further complication in giving a complete mathematical characterization of possible bases $B_{3}$ that support elliptically fibered CalabiYau fourfolds, which requires more sophisticated mathematics than the classification of bases $B_{2}$ for threefolds. Notwithstanding the challenges of finding a complete classification of fourfolds for realistic F-theory models, this approach can give a broad class of models with semi-realistic phenomenological features, and all F-theory constructions of physical theories with standard model-like features must be describable in this general framework.

We now describe some more detailed aspects of each of the steps above in turn.

\subsection{Choose a base $B_{3}$}

A broad class of bases $B_{3}$ that can support $4 \mathrm{D}$ F-theory models are known. Many CalabiYau fourfolds that arise as hypersurfaces or complete intersections of toric varieties have been studied using the original approach of Batyrev [38, 39] (see for example [40, 41]); 
many of these fourfolds have a description as an elliptic fibration over an appropriate toric base $B_{3}$ [42] and are appropriate for F-theory compactifications [43-47]. A complete classification of all bases $B_{3}$ that have the form of $\mathbb{P}^{1}$ bundles over a complex surface $B_{2}$ and that support elliptically fibered Calabi-Yau fourfolds giving F-theory models with smooth heterotic dual constructions was given in [22]; in these cases the base $B_{2}$ is a generalized del Pezzo surface. A much broader class of bases $B_{3}$ can be constructed as $\mathbb{P}^{1}$ bundles over surfaces $B_{2}$ that can act as bases for elliptically fibered threefolds; a complete list of all of the over 100,000 toric and "semi-toric" surfaces $B_{2}$ of this type has been constructed $[48,49]$, and a systematic analysis of $\mathbb{P}^{1}$ bundles over such bases will appear elsewhere [50]. Most of the explicit examples considered later in this paper use bases $B_{3}$ of this form. Recent constructions of Calabi-Yau fourfolds as complete intersections, of which more than $99.9 \%$ satisfy appropriate conditions for elliptic fibration structure [51, 52], also promise to provide a rich supply of examples.

Following the framework of Mori theory [53], an even broader class of base threefolds $B_{3}$ can in principle be constructed, though unlike the case of Calabi-Yau threefolds, where all minimal bases are known [54], a complete set of minimal threefold bases $B_{3}$ from which all others can be constructed by suitable geometric transitions is not yet known. For Calabi-Yau threefolds, the set of allowed bases $B_{2}$ is connected by blowing up and down points on the base, corresponding to tensionless string transitions $[3,14]$ in the associated $\mathrm{F}$ theory models. While the bases $B_{3}$ for elliptically fibered Calabi-Yau fourfolds are similarly connected through birational (blow-up and blow-down) transitions, it is not known if the total space of $B_{3}$ 's has only one or more connected components.

\subsection{Check for non-Higgsable clusters}

Having chosen a base $B_{3}$, the next step is to consider the general Weierstrass model over that base, and determine whether or not there are non-Higgsable clusters. There are a number of ways of doing this, depending on the geometry of the base. When the base is toric, this computation is straightforward. Toric bases can be described either using a gauged linear sigma model language common in physics (see e.g. [55]), or equivalently in terms of a toric fan as is standard in mathematics [56]; in toric cases there is a straightforward algorithm for determining all monomials in the Weierstrass coefficients $f, g$ through solutions to a given set of inequalities, and one can check directly the order of vanishing on each of the toric divisors, as described for example in [48].

There are also systematic methods that can be applied when the base is not toric. When the base is a surface $B_{2}$, the divisor class $[-K]$ can be formally decomposed over $\mathbb{Q}$ into irreducible components; this Zariski decomposition can be carried out using the intersection properties of curves on the surface, and determines the minimal degrees of vanishing of $f, g$ on any curves in the base. This was the approach used in [9] to identify all non-Higgsable clusters in six dimensions. When the base is a general non-toric threefold $B_{3}$ the analogue of the Zariski decomposition is somewhat more complicated to describe in terms of the intersection numbers on the threefold, but a related method of analysis in terms of the geometry of divisors and curves can determine the presence of non-Higgsable clusters. An explicit way to determine minimal vanishing degrees of $f, g$ on a divisor in 
a general base threefold using information about the line bundles over the divisor that contain the coefficients in an expansion of $f, g$ around that divisor is developed in $[22,23]$.

For concreteness, we present here a simple example that demonstrates the appearance of a non-Higgsable cluster using the gauged linear sigma model approach to toric geometry that may be most familiar to physicists. For the examples described later in the paper, we primarily use the fan description of toric varieties and/or the more abstract description of the line bundles in which the Weierstrass coefficients take values. All of these approaches can be used in more complicated examples and may be easily analyzed on a computer.

Consider the possibility

$$
B_{3}=\mathbb{P}^{1} \times \mathbb{F}_{8}
$$

where $\mathbb{F}_{8}$ is the eighth of the infinite series of Hirzebruch surfaces $\mathbb{F}_{n}$ that themselves describe $\mathbb{P}^{1}$ bundles over $\mathbb{P}^{1}$. This base $B_{3}$ is a toric variety and can be described as a quotient space using the following coordinates and $\mathbb{C}^{*}$ actions

\begin{tabular}{|l|cccccc|}
\hline & $x_{1}$ & $x_{2}$ & $x_{3}$ & $x_{4}$ & $x_{5}$ & $x_{6}$ \\
\hline $\mathbb{C}_{1}{ }^{*}$ & 1 & 1 & & & & \\
\hline $\mathbb{C}_{2}{ }^{*}$ & & & 1 & & 1 & 8 \\
\hline $\mathbb{C}_{3}{ }^{*}$ & & & & 1 & & 1 \\
\hline
\end{tabular}

where empty entries are zero. We also define the set

$$
S=\left\{x_{1}=x_{2}=0\right\} \cup\left\{x_{3}=x_{5}=0\right\} \cup\left\{x_{4}=x_{6}=0\right\} .
$$

Then $B_{3}$ is defined by taking the $\left(x_{1}, \ldots, x_{6}\right) \in \mathbb{C}^{6}$, removing $S$, and quotienting by the $\mathbb{C}^{*}$ actions

$$
B_{3}=\frac{\mathbb{C}^{6} \backslash S}{\mathbb{C}_{1}^{*} \times \mathbb{C}_{2}^{*} \times \mathbb{C}_{3}^{*}},
$$

where the $\mathbb{C}^{*}$ actions give equivalence classes; for example, the $\mathbb{C}_{2}^{*}$ quotient identifies

$$
\left(x_{1}, \ldots, x_{6}\right) \sim\left(x_{1}, x_{2}, \lambda x_{3}, x_{4}, \lambda x_{5}, \lambda^{8} x_{6}\right) \lambda \in \mathbb{C}^{*}
$$

and similarly for the other actions. In the quotient space, $\left(x_{1}, \ldots, x_{6}\right)$ are homogeneous coordinates. Such a quotient space can arise as the set of supersymmetric ground states of an appropriate gauged linear sigma model.

Having defined $B_{3}$ in this example, we may now construct the most general Weierstrass model $(2.1)$. Here $\mathcal{O}\left(-K_{B}\right)=\mathcal{O}(2,10,2)$, meaning that sections of the line bundle associated with the divisor $-K_{B}$ transform with the given powers of the transformation parameters under the three $\mathbb{C}^{*}$ actions; this result is obtained by taking the sum of the charges of the coordinates under the $\mathbb{C}^{*}$ actions. It follows that $f$ and $g$ are global sections of the line bundles

$$
f \in \mathcal{O}(8,40,8) \quad g \in \mathcal{O}(12,60,12) .
$$

Each monomial in $f$ therefore scales with degrees 8,40 , and 8 under $\mathbb{C}_{1}^{*}, \mathbb{C}_{2}^{*}$, and $\mathbb{C}_{3}^{*}$, respectively, and similarly for monomials in $g$. Furthermore, the exponent of each monomial must be non-negative. The monomial

$$
x_{1}^{3} x_{2}^{5} x_{3}^{5} x_{4}^{4} x_{5}^{3} x_{6}^{4},
$$


for example, scales as a monomial in $f$. It is a simple exercise to determine all such allowed monomials in $f$, and thereby to determine the most general $f$. A similar computation with different scaling degrees holds for $g$.

Note the following interesting feature, however: $f$ may not contain a monomial $x_{6}^{n}$ for $n>5$ since it would oversaturate the scaling degree of $\mathbb{C}_{2}^{*}$ and the powers of $x_{i}$ (in particular $x_{4}$ ) in any monomial must be non-negative. Thus $n$ must satisfy $n \leq 5$ and therefore every monomial in $f$ must have $x_{4}^{m}$ with $m \geq 3$ in order to saturate the scaling degree of $\mathbb{C}_{3}^{*}$. A similar analysis for $g$ shows that the monomials in $f, g$ can always be written in the form

$$
f=x_{4}^{3} \tilde{f} \quad g=x_{4}^{5} \tilde{g} .
$$

We see that the most general $f$ and $g$ have overall factors of $x_{4}$, where $x_{4}=0$ defines a divisor in $B_{3}$. This is the characteristic signature of a non-Higgsable cluster, and from table 1 we identify that there is an $E_{7}$ non-Higgsable cluster along $x_{4}=0$. Note that for simplicity we chose an example where outer monodromy would not be in effect.

\subsection{Ensure the existence of $\mathrm{SU}(3) \times \mathrm{SU}(2)$}

At this point in the systematic process we are describing we have chosen a base $B_{3}$ and have identified all non-Higgsable clusters exhibited by the generic Weierstrass model over $B_{3}$. If there are $\mathrm{SU}(3)$ or $\mathrm{SU}(2)$ non-Higgsable gauge group factors we do not have to specialize in moduli space to ensure their existence.

We would now like to discuss broadly the possible ways to realize the non-abelian sector of the standard model. Specifically, if the gauge group of the four-dimensional compactification is to contain the subgroup $G_{32}=\mathrm{SU}(3) \times \mathrm{SU}(2)$, this group must either arise from a non-Higgsable cluster, or the Weierstrass model must be tuned to realize whatever part of this group is not found through a non-Higgsable cluster. Furthermore, for quarklike matter to arise that is charged under both factors, the divisors supporting these two gauge group factors must intersect in a curve in $B_{3}$. There are several logical possibilities:

i) No non-Higgsable gauge group. In this case the Weierstrass model must either be tuned to contain both the $\mathrm{SU}(3)$ and $\mathrm{SU}(2)$ gauge group factors on intersecting divisors, or to have have a grand unified gauge factor on a single non-rigid divisor that contains $\mathrm{SU}(3) \times \mathrm{SU}(2)$ as a subgroup.

ii) Non-Higgsable $\mathbf{S U}(2)$. In this case either an additional SU(3) must be tuned or the original $\mathrm{SU}(2)$ must be enhanced to an $\mathrm{SU}(3)$ and an additional $\mathrm{SU}(2)$ tuned on an intersecting divisor.

iii) Non-Higgsable SU(3). An additional $\mathrm{SU}(2)$ must be tuned on a divisor that intersects the divisor carrying the $\mathrm{SU}(3)$.

iv) Non-Higgsable $\mathbf{S U}(2) \times \mathbf{S U}(2)$. If there is a non-Higgsable $\mathrm{SU}(2) \times \mathrm{SU}(2)$ on two intersecting divisors, the Weierstrass model must be tuned so that one of them is enhanced to $\mathrm{SU}(3)$. 
v) Non-Higgsable $\mathbf{S U}(3) \times \mathbf{S U}(2)$. In this case the entire desired nonabelian gauge group is automatically present in the generic Weierstrass model over $B_{3}$ and no further tuning is necessary.

In almost all cases considered previously in the literature, the approach taken is that of case i). This is the only approach possible when the gauge group factors $\mathrm{SU}(3) \times \mathrm{SU}(2)$ can be realized geometrically in an F-theory construction within a unifying group $\mathrm{SU}(5)$, so that the $\mathrm{SU}(3)$ and $\mathrm{SU}(2)$ factors are realized on divisors in the same homology class, since $\mathrm{SU}(5)$ is associated with a type $I_{5}$ singularity, which cannot arise on any divisor supporting a non-Higgsable cluster. For $\mathrm{SO}(10)$ and $E_{6}$ unification scenarios, the set of possibilities is somewhat richer. $E_{6}$ can be realized through a non-Higgsable cluster, and could then be broken down to a standard model gauge group through a flux on the sevenbrane world volume. While $\mathrm{SO}(10)$ cannot be realized through a non-Higgsable cluster, a divisor with a non-Higgsable type $I_{0}^{*}$ singularity (or a type $I I I$ or $I V$ singularity) could be enhanced to $\mathrm{SO}(10)$, and in principle this group could be broken down to $\mathrm{SU}(3) \times \mathrm{SU}(2)$ in such a way that part of the gauge group was still non-Higgsable. Approaches to GUT constructions using approach i) have been extensively studied in the literature, beginning with [57-59], and extending to global constructions [60-66]; for reviews see [7, 8]. In much of this work, internal flux on the seven-branes is the mechanism used for GUT breaking, so while these investigations have mostly focused on constructions of type i), an extension to include non-Higgsable structures within $\mathrm{SO}(10)$ or $E_{6}$ models may be natural. In some recent work, such as $[67,68]$, approach i) has been taken but without the GUT assumption, so that the divisors supporting the $\mathrm{SU}(3)$ and $\mathrm{SU}(2)$ factors are assumed to be distinct and intersecting. These constructions are closer in spirit to those we consider in the rest of this paper; the difference is that the constructions we focus on here correspond to cases ii)-v).

In all cases other than i) above, at least one of the nonabelian gauge group factors is realized through a non-Higgsable cluster. One of the primary points of this paper is that the other possibilities can be realized naturally in F-theory and offer some interesting phenomenological features. We focus in particular on the cases iii) and v), where the SU(3) nonabelian factor is non-Higgsable, motivated by the observed fact that the SU(3) of QCD observed in nature is unbroken. As we show in section 5, such non-Higgsable clusters can arise in specific simple examples of base threefolds $B_{3}$. Note that while a single nonHiggsable SU(3) or SU(2) factor can easily arise in an F-theory model with a smooth dual heterotic description, if there is a non-Higgsable $\mathrm{SU}(3)$ and $\mathrm{SU}(2)$ is realized on a separate intersecting divisor, this would correspond to a singular geometry in any dual heterotic description. Thus, the cases iii) and v), of a non-Higgsable QCD SU(3) group, are most clearly visible from geometry in the F-theory approach.

The classification of $\mathrm{SU}(3)$ and $\mathrm{SU}(2)$ factors above is according to whether or not they arise from non-Higgsable groups, but for each of these gauge group factors, a more refined set of cases can be distinguished based on the precise Kodaira singularity types that realize each factor. As discussed earlier, both $\mathrm{SU}(3)$ and $\mathrm{SU}(2)$ can be realized in different ways through different Kodaira singularities that involve different numbers of seven-branes. A detailed list of the possibilities is given in table 3. Some further comments 


\begin{tabular}{|cccc|}
\hline & $\exists$ Higgsable & $\exists$ Non-Higgsable & \# Branes \\
\hline $\mathrm{SU}(3)$ from $I_{3}$ & Yes & No & 3 \\
$\mathrm{SU}(3)$ from $I V$ & Yes & Yes & 4 \\
\hline $\mathrm{SU}(2)$ from $I_{2}$ & Yes & No & 2 \\
$\mathrm{SU}(2)$ from $I I I$ & Yes & Yes & 3 \\
$\mathrm{SU}(2)$ from $I V$ & Yes & Yes & 4 \\
\hline
\end{tabular}

Table 3. Distinct realizations of $\mathrm{SU}(3)$ and $\mathrm{SU}(2)$ in F-theory through different Kodaira singularity types, together with the number of seven-branes realizing the gauge theory and whether or not there exist Higgsable and non-Higgsable configurations.

on the table may be relevant. First, only the $I_{3}$ and $I_{2}$ realizations exist at weak coupling and these cannot be non-Higgsable; the states realizing the others are non-trivial $(p, q)$ string junctions associated with type $I I I$ or type $I V$ singularities, which can either be Higgsable or non-Higgsable. Second, the $\mathrm{SU}(2)$ realization involving a type $I V$ fiber is an $\mathrm{Sp}(1) \cong \mathrm{SU}(2)$ realization where there is outer monodromy on the type $I V$ fiber.

The case v) is associated with a non-Higgsable cluster that arises when there are forced type III and IV Kodaira type singularities on intersecting divisors, or two intersecting type IV singularities where one has monodromy giving an $\mathrm{SU}(2)$. A particularly interesting aspect of the first of these geometries is that the minimal $(1,2,3)$ and $(2,2,4)$ vanishing degrees of $f, g, \Delta$ on the divisors carrying the gauge group factors force vanishing degrees of at least $(3,4,8)$ on the intersection curve. Here the number of seven-branes, associated with the degree of vanishing of $\Delta$, is increased to 8 rather than $3+4=7$, since ord $(\Delta) \geq \max (3 \operatorname{ord}(f), 2 \operatorname{ord}(g))$. With a generalization of the results of [29], as we describe below, the resulting matter is that associated with the embedding of $\mathrm{SU}(3) \times \mathrm{SU}(2)$ in the adjoint of $E_{6}$, with interesting phenomenological properties.

Note that in all cases that we consider here, the intersection between the divisors supporting the $\mathrm{SU}(3)$ and $\mathrm{SU}(2)$ factors is assumed to be transverse. Other constructions, for example where the divisors are tangent at the intersection point, could also be investigated.

\subsection{Identify a configuration with a $U(1)$ factor}

We have identified the possible ways to engineer $\mathrm{SU}(3) \times \mathrm{SU}(2)$ in F-theory, but have not yet addressed the $\mathrm{U}(1)$ factor in $G_{321}$. If $G_{32}$ arises as a subgroup of some grand unified group $G_{G U T}$, the weak hypercharge $\mathrm{U}(1)_{Y}$ may arise from the the non-abelian structure of $G_{G U T}$. If not, $\mathrm{U}(1)_{Y}$ must arise from a non-trivial Mordell-Weil group of sections of the elliptic fibration. The number of $\mathrm{U}(1)$ factors is given by the rank of the Mordell-Weil group [3], and a realistic theory requires that one may be identified as the weak hypercharge.

In general, the problem of identifying the Mordell-Weil group is a difficult mathematical problem. Much recent work has focused on F-theory models with one or more U(1) factors, and while there is some understanding of models with small Mordell-Weil rank, a general understanding of the geometry of models with generic Mordell-Weil rank is still lacking. In the case of a single $\mathrm{U}(1)$ factor, however, there is a relatively clear understanding of 
how models can be constructed. The generic form of a Weierstrass model for a Calabi-Yau elliptic fibration with a section corresponding to Mordell-Weil rank one or higher is given in [69]. We use this approach in section 6.1 to describe some aspects of the tuning of an additional $\mathrm{U}(1)$ factor in models with a non-Higgsable $\mathrm{SU}(3) \times \mathrm{SU}(2)$ gauge group. In the recent works $[67,68]$ progress was made in realizing the weak hypercharge in F-theory by engineering a non-trivial Mordell-Weil group, though as mentioned above those analyses did not utilize non-Higgsable clusters.

\subsection{Low-energy physics, matter, and G-flux}

As mentioned at the beginning of this section, the preceding discussion is carried out at the level of pure geometry. While in six-dimensional theories the spectrum of massless states in a low-energy supergravity theory is simply related to the geometric structure of the associated Calabi-Yau threefold, for 4D theories there are additional effects which may or may not modify the geometric gauge group. G-flux and possible other effects such as D3-branes can modify both the geometric gauge group and matter content of the theory.

On the one hand, G-flux can correspond to flux in the world-volume of a seven-brane, which can break a geometrically non-Higgsable gauge group factor. On the other hand, the superpotential induced by G-flux can push the theory to a special sublocus of moduli space where the symmetry is enhanced beyond the geometric gauge group obtained a generic point in complex structure moduli space. G-flux can also affect the matter content of the theory. The geometric analysis captures the Lie algebraic structure of possible matter states, but only describes non-chiral $\mathcal{N}=2$ type matter. G-flux can modify the matter spectrum, producing chiral matter fields in the various representations identified through geometry. While in this paper we focus on the geometric structures involved, in section 7.2 we utilize an anomaly analysis to determine the minimal chiral $\mathrm{SU}(3) \times \mathrm{SU}(2) \times \mathrm{U}(1)$ which may be realized in the non-Higgsable QCD scenario we propose. In general, though, a full understanding of any realistic realization of the standard model spectrum in F-theory will require a detailed analysis of the role of G-flux, the superpotential, and the chiral spectrum. We describe some further details of the issues involved in section 6.2.

\section{Non-Higgsable QCD}

In the last sections we have studied the possible ways to realize the standard model in F-theory. We have also seen that F-theory exhibits special realizations of $\mathrm{SU}(3)$ and $\mathrm{SU}(2)$ gauge theories on seven-branes that do not exist for other $\mathrm{SU}(n)$, and furthermore that these realizations are the only $\mathrm{SU}(n)$ realizations in F-theory that may be non-Higgsable. Given these facts and the existence of an unbroken QCD sector in nature, in this and the following sections we will focus in particular on the possibility of realizing $\mathrm{SU}(3)_{Q C D}$ via a non-Higgsable seven-brane.

To consider such a possibility, we will need to recall the possible realizations of $\mathrm{SU}(n)$ gauge theories in F-theory.

1. $\mathrm{SU}(n)$ may be realized by an $I_{n}$ fiber for any $n$, but these are necessarily Higgsable and are the F-theory lift of $n$ coincident $D 7$-branes. 
2. $\mathrm{SU}(3)$ may also be realized via a type $I V$ fiber without outer monodromy. This configuration utilizes four seven-branes and does not admit a weakly coupled type IIB description, as it necessarily involves non-trivial $(p, q)$ string junctions. Such a configuration could potentially be non-Higgsable, but is not required to be.

3. SU(2) may also be realized via a type $I I I$ fiber without outer monodromy, or a type $I V$ fiber with outer monodromy. Both could potentially be non-Higgsable, but are not required to be.

We find it suggestive that $\mathrm{SU}(3)$ and $\mathrm{SU}(2)$ are the only gauge factors that may be nonHiggsable.

Given these facts, we see there are three qualitatively different ways to realize nonHiggsable QCD. This follows for a simple reason: there is one possible realization of a non-Higgsable $\mathrm{SU}(3)$, namely from a seven-brane associated to a type $I V$ singular fiber, but it may be paired with any of three possible realizations of $\mathrm{SU}(2)$. These possibilities are realized by seven-branes associated to a type $I V$ fiber with outer monodromy, a type $I I I$ fiber, or an $I_{2}$ fiber. We therefore refer to these realizations of non-Higgsable QCD as $I V-I V m, I V-I I I$, and $I V-I_{2}$, respectively, according to their realization of the standard model gauge subgroup $\mathrm{SU}(3) \times \mathrm{SU}(2)$. We will focus slightly more on the $I V$ - III realization since the spectrum at the $I V-I I I$ intersection is most interesting.

We emphasize from the outset that in this classification of the three realizations of non-Higgsable QCD, we have not made any assumptions as to whether or not the $\mathrm{SU}(2)$ factor is also non-Higgsable. Though the $I V-I_{2}$ case always has a Higgsable $\mathrm{SU}(2)$, we will see in examples that either a Higgsable or non-Higgsable $\mathrm{SU}(2)$ are possible in the $I V$ - $I I I$ and $I V-I V m$ cases.

In studying non-Higgsable QCD in F-theory models, we find it helpful to use a variety of techniques, in part because each has different advantages. In this section we review the string junction description of $\mathrm{SU}(3)$ and $\mathrm{SU}(2)$ seven-branes realized by type $I V$ and $I I I$ fibers, respectively. We also present the first junction analysis of the geometry near a $I V$ III collision and discuss the implications for matter spectra. One feature of the junction analysis it that it demonstrates the possible existence of $\mathrm{SU}(3) \times \mathrm{SU}(2)$ singlet states in the $I V-I I I$ realization, which may be interpreted as right-handed electrons or neutrinos in some models.

In analyzing the F-theory geometry of the various non-Higgsable QCD models we generally begin by studying the gauge group and then consider the matter content at the level of Lie algebra representations realized in the geometry. The different constructions of the gauge group have already been reviewed in the previous sections, and the junction analysis gives a deeper perspective on the role of seven-branes in these different geometries.

The analysis of the matter content is somewhat more complicated. There are a number of ways to analyze matter representations associated with codimension two singularities in F-theory. The original Katz-Vafa analysis [29] of the simplest types of codimension two singularities - namely those where the Kodaira type of the codimension two singularity has a rank one greater than that of the generic point in the codimension one locus - involves a straightforward decomposition of the adjoint representation of the larger 
group; even there, however, some care must be taken in determining the multiplicity of each representation. For 6D theories, anomaly cancellation [70-73] provides a powerful tool that in many cases uniquely determines the representation content of a given theory, including multiplicities [30, 33, 37, 74, 75], though this approach does not detect singlet representations of the nonabelian gauge group that can play an important role, for example, in realizations of the standard model. As discussed in section 2, for $4 \mathrm{D}$ theories with more complicated singularities a resolution of the singularity can be achieved by standard methods in algebraic geometry, and more recently the method of deformation has been developed as a useful alternative to analyzing such singularities.

We focus in this section on the deformation method using junctions and in the next section on $6 \mathrm{D}$ anomaly analyses. Since a number of factors are relevant in determining the multiplicity and chirality of matter fields in a 4D theory, including the genus of the matter curve, G-flux, and possibly other considerations, our main intent here is to determine simply the set of allowed representations, and we do not attempt to carry out a precise calculation of multiplicity and chirality in specific 4D models.

In the next section we present a number of examples. This has two purposes. First, in four-dimensional theories we describe explicit geometries that realize $\mathrm{SU}(3) \times \mathrm{SU}(2)$ non-Higgsable clusters and SU(3) non-Higgsable clusters with Higgsable SU(2) factors, in both cases with matter; this is an existence proof for constructions of these types. Second, by studying six-dimensional theories where we engineer the $I V-I V m, I V-I I I$, and $I V-I_{2}$ intersections, we determine the set of nonzero charges that can arise in the spectrum using anomaly cancellation. Specifically, anomaly cancellation allows us to precisely determine the non-trivial $\mathrm{SU}(3) \times \mathrm{SU}(2)$ matter representations localized at the collision of the $\mathrm{SU}(3)$ and $\mathrm{SU}(2)$ seven-branes, including multiplicities in the $6 \mathrm{D}$ theory, and we can match the set of representations found in that way with the junction analysis of this section.

Of the three ways $\left(I I I, I V m, I_{2}\right)$ that a $\mathrm{SU}(2)$ can be realized in conjunction with a non-Higgsable $\mathrm{SU}(3)$, in this section we focus on the $I V-I I I$ intersection. The other possibilities are analyzed in the following section. A summary of the geometric matter realized in these three configurations is given in section 7.1.

\subsection{Non-perturbative realizations of $\mathrm{SU}(3)$ and $\mathrm{SU}(2)$}

We begin by reviewing the structure of $\mathrm{SU}(2)$ and $\mathrm{SU}(3)$ gauge states that arise from a type $I I I$ and type $I V$ fiber, as described by string junctions [24-26] since they differ significantly from the case of $n$ D7-branes. See [28] for detailed deformations that give rise to this data. These junction descriptions are well understood but set the stage for the more complicated junction descriptions of intersecting seven-branes.

Before reviewing these $\mathrm{SU}(3)$ and $\mathrm{SU}(2)$ theories, we present a brief basic review of string junctions and their connection to deformations of algebraic varieties as developed ${ }^{3}$ in $[27,28]$. Consider an M-theory compactification to $(d-1)$ dimensions on an elliptically

\footnotetext{
${ }^{3}$ For computer codes which aid in performing these computations, see http://files.jhhalverson.com/deformations.
} 
fibered Calabi-Yau manifold $X$ in Weierstrass form

$$
y^{2}=x^{3}+f x+g=: v_{3}(x) .
$$

This theory becomes an F-theory compactification to $d$ dimensions in its vanishing fiber limit $X \rightarrow X_{v}$. Suppose that $X$ exhibits a codimension one singular fiber at a locus $z=0$ where a local coordinate $z$ on the base vanishes, giving a non-abelian gauge factor $G$, so that $\Delta=z^{n} \Delta_{r}$ for some $n>2$. Then a small disc transverse to $z=0$ defines an (open) elliptic surface in $X$; this surface comes in a family that can be parameterized by local complex coordinates on the locus $\{z=0\}$. If one performs a small deformation in a local patch by deforming $f$ and $g$ such that $X \rightarrow X_{\epsilon}$ has discriminant $\Delta=z^{n} \Delta_{r}+\epsilon \Delta_{\epsilon}$ with $\epsilon \in \mathbb{C}$ and $z$ not dividing $\Delta_{\epsilon}$, then the non-abelian gauge symmetry along $z=0$ in $X$ is spontaneously broken. By taking the limit $\epsilon \rightarrow 0$ the symmetry is restored.

Consider a generic elliptic surface in the family of elliptic surfaces that cross the singular locus $z=0$ in $X$. In performing the deformation $X \rightarrow X_{\epsilon}$ for small $\epsilon$, the $n$ marked points at $z=0$ in the disc $D$ (which are seven-branes in the F-theory limit) spread out into $n$ non-degenerate marked points $z_{i}$, each with an $I_{1}$ singular fiber above it in the elliptic surface. Each such fiber has an associated vanishing cycle, which is the $(p, q)$ label of the seven-brane at $z_{i}$ in F-theory. The fiber above $z=0$ in the deformed elliptic surface is smooth, and by following straight line paths from $z=0$ to the $z_{i}$, the vanishing cycles can be read off systematically. Specifically, for any point in $D \backslash\left\{z_{i}\right\}$ the associated fiber is smooth and $v_{3}(x)$ has three non-degenerate roots that might appear as the dots in

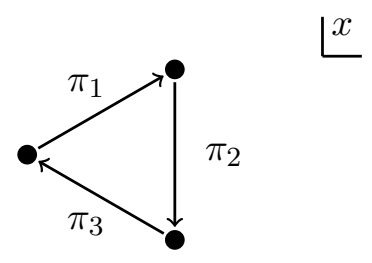

These points are where the double cover $y^{2}=v_{3}(x)$ degenerates. A one-cycle on the elliptic fiber can be defined by following a path from one dot to another, going to the other sheet, and then following a path back to the original dot; in the figure we have defined three one-cycles $\pi_{1}, \pi_{2}, \pi_{3}$ subject to the condition $\pi_{1}+\pi_{2}+\pi_{3}=0$. In following the mentioned straight line path from $z=0$ to one of the $z_{i}$, two of these points will collide as $z \rightarrow z_{i}$ and the corresponding one-cycle vanishes in this limit. Picking a basis, this determines the $(p, q)$ label of the seven-brane at $z_{i}$, and in particular one can identify an ordered set of vanishing cycles $Z=\left\{\pi_{1}, \cdots, \pi_{n}\right\}$.

As expected from string theory, objects can stretch between the branes located at the $z_{i}$. Suppose that two branes at $z_{1}$ and $z_{2}$ have the same vanishing one-cycle $\pi_{1}=\pi_{2} \equiv \pi$. Then in following a path from $z_{1}$ to $z_{2}$, the vanishing one-cycle begins as a point at $z_{1}$, grows, shrinks, and then finally collapses to a point at $z_{2}$; this defines a two-sphere in the total space of the elliptic surface with one leg on the fiber and one leg on the base, and an M2-brane can be wrapped on this two-cycle in the M-theory picture. In the F-theory limit this M2-brane becomes a fundamental string (with an appropriate choice of $\mathrm{SL}(2, \mathbb{Z})$ 
frame). If there were three seven-branes, it is natural to represent the state from $z_{1}$ to $z_{2}$ as $(1,-1,0)$, and the negative of this for the state from $z_{2}$ to $z_{1}$.

More generally, though, if a collection of branes at the $z_{i}$ have different vanishing cycles, it is possible to form two-spheres that end on multiple branes, with an associated "junction" in the base that generalizes the path from one $z_{i}$ to another. We will see what this means in an example, momentarily; for now just note that we represent such a junction by a tuple $\left(J_{i}\right)$ in $\mathbb{Z}^{n}$ where $n$ is the number of branes being analyzed in the deformation and $J_{i}$ is the number of "prongs" coming off the brane at $z_{i}$. A junction $J$ may have a loose end that goes off to infinity with some asymptotic charge $a(J) \equiv \sum J_{i} \pi_{i}$, which is just the $(p, q)$ charge of the loose end (the one-cycle wrapped by an M2-brane in the M-theory picture) emanating to infinity (perhaps ending on a far away brane). Junctions with $a(J)$ zero end entirely within the branes at the $z_{i}$, each defining a two-sphere on which M2-branes can be wrapped in the M-theory picture; these become string junctions in the F-theory limit. One definition of a string junction, then, is the object obtained by taking the F-theory limit of an M2-brane on the deformation two-cycle. Finally, the two-spheres associated to junctions have non-trivial topological intersections in the elliptic surface. In [27] we wrote down a formula for these intersections, which determines a so-called $I$-matrix that conveniently computes the intersections.

From this data there are various computations that can be performed. For example, if the deformed branes are associated to a Kodaira singularity with Lie algebra $G$ (which may or may not be the gauge group, depending on details), then the junctions with $a(J)$ zero and self-intersection -2 are determine the root lattice of $G$.

Having reviewed the basics of junctions, let us turn to examples, which may provide further clarification.

SU(2) from a type III fiber and Mercedes W-bosons. A seven-brane with gauge symmetry $\mathrm{SU}(2)$ can arise from a type $I I I$ fiber; if it is along $z=0$ and specified by a Weierstrass model, then $(\operatorname{ord}(f), \operatorname{ord}(g), \operatorname{ord}(\Delta))=(1,2,3)$. Note that since $\operatorname{ord}(\Delta)=3$, this $\mathrm{SU}(2)$ gauge symmetry arises when three seven-branes collide, as opposed to the common $\left(I_{2}\right)$ case in weakly coupled type IIB that arises when two D7-branes collide. In [28] it was shown that the type $I I I$ configuration can arise from three seven-branes with $(p, q)$ labels

$$
Z_{I I I}=\left\{\pi_{2}, \pi_{1}, \pi_{3}\right\}
$$

which has associated $I$-matrix

$$
I=(\cdot, \cdot)=\left(\begin{array}{ccc}
-1 & 1 / 2 & -1 / 2 \\
1 / 2 & -1 & 1 / 2 \\
-1 / 2 & 1 / 2 & -1
\end{array}\right)
$$

The topological self intersections of junctions can be determined from this matrix. Defining the asymptotic charge $a(J)=\sum J_{i} \pi_{i} \in H_{1}\left(T^{2}, \mathbb{Z}\right)$, the set $R=\left\{J \in \mathbb{Z}^{3} \mid(J, J)=-2\right.$ and $\left.a(J)=0\right\}$ is computed to be $R=\{(1,1,1),(-1,-1,-1)\}$. 
These are three-pronged string junctions
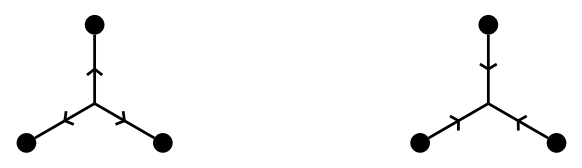

that are the $W_{+}$and $W_{-}$bosons of $\mathrm{SU}(2)$, where the charge is with respect to the Cartan U(1) of SU(2); in the M-theory picture one dimension lower these are M2-branes wrapped on the corresponding two-cycles. In [28] it was shown how to build up higher spin representations of $\mathrm{SU}(2)$ using this junction data.

SU(3) from a type $\boldsymbol{I} \boldsymbol{V}$ fiber. A seven-brane with gauge symmetry SU(3) can arise from a type $I V$ fiber; if it is along $z=0$ and specified by a Weierstrass model, then $(\operatorname{ord}(f), \operatorname{ord}(g), \operatorname{ord}(\Delta))=(2,2,4)$. Again we see that there are more seven-branes than expected from type IIB expectations; in the perturbative IIB case, $\mathrm{SU}(3)$ arises from a stack of three coincident D7-branes, i.e. from a type $I_{3}$ fiber, whereas for a type $I V$ fiber $\mathrm{SU}(3)$ is realized by four seven-branes. In [28] it was shown that this type $I V$ configuration can arise from four seven-branes with $(p, q)$ labels

$$
Z_{I V}=\left\{\pi_{1}, \pi_{3}, \pi_{1}, \pi_{3}\right\} .
$$

and associated $I$-matrix

$$
I=(\cdot, \cdot)=\left(\begin{array}{cccc}
-1 & 1 / 2 & 0 & 1 / 2 \\
1 / 2 & -1 & -1 / 2 & 0 \\
0 & -1 / 2 & -1 & 1 / 2 \\
1 / 2 & 0 & 1 / 2 & -1
\end{array}\right)
$$

Computing (as we did for the type $I I I$ case) the set of junctions with self-intersection -2 and asymptotic charge zero, we obtain

$$
\begin{aligned}
& \{(-1,-1,1,1),(-1,0,1,0),(0,-1,0,1), \\
& \quad(0,1,0,-1),(1,0,-1,0),(1,1,-1,-1)\},
\end{aligned}
$$

a set of six string junctions that fills out (together with the Cartan elements, which are two copies of $(0,0,0,0))$ an adjoint of $\mathrm{SU}(3)$. See [28] for a detailed description of simple roots and how to build arbitrary $\mathrm{SU}(3)$ representations using this junction data.

\section{2 $\mathrm{SU}(3) \times \mathrm{SU}(2)$ from a $I V-I I I$ collision}

We would like to study the geometry and physics at the $I V$-III intersection; we are interested in particular in the matter spectrum that occurs there. In a simple $I_{3} \times I_{2}$ collision, the matter fields consist simply of bifundamental matter that transforms in the $(\mathbf{3}, \mathbf{2})$ of $\mathrm{SU}(3) \times \mathrm{SU}(2)$, associated with open strings that connect the three D7-branes in the $I_{3}$ to the two D7-branes in the $I_{2}$. We expect to find a similar $(\mathbf{3}, \mathbf{2})$ matter representation in other constructions of $\mathrm{SU}(3) \times \mathrm{SU}(2)$ on intersecting divisors. If these gauge groups are 
those of the standard model, then the natural interpretation of the $(\mathbf{3}, \mathbf{2})$ matter is as the left-handed quark doublets.

The surprising feature of the $I V-I I I$ intersection is that we also get fields that transform in the same representations as the other matter fields of the standard model. Note that the purely geometric analysis always describes non-chiral ( $\mathcal{N}=2$ ") matter, so that for every representation such as $(\mathbf{3}, \mathbf{2})$ there is a matter field of opposite chirality in the conjugate representation $(\overline{\mathbf{3}}, \overline{\mathbf{2}})$ (though $\overline{\mathbf{2}}=\mathbf{2}$ ). In much of the ensuing discussion we do not explicitly mention these conjugate representations, assuming throughout that all matter arising from purely geometric analyses is non-chiral. The reduction through G-flux to chiral matter is discussed in section 6 .

We first review the structure of matter realized at a perturbative $\mathrm{SU}(3) \times \mathrm{SU}(2)$ intersection realized by intersecting D7-branes. We then present a string junction analysis in neighborhood of the collision; this is not a full deformation analysis of string junctions but gives intuition for why the states localized at the collision are richer than in the perturbative case. We determine the precise charged $\mathrm{SU}(3) \times \mathrm{SU}(2)$ spectrum at a $I V$-III collision in the next section via a six-dimensional anomaly analysis. Combining these analyses gives us a complete list of the kinds of representations that can appear at this intersection. As mentioned above, a complete treatment of multiplicities and chirality would require a more thorough analysis that we leave for later work. Note, however, that the analysis of this section applies to the matter localized at any $I V$ - $I I I$ collision, regardless of Higgsability or non-Higgsability and independent of dimension.

"Quark doublets" from an $\boldsymbol{I}_{\mathbf{3}}-\boldsymbol{I}_{\mathbf{2}}$ collision. We begin with the open string/string junctions description of a well known case that will be in sharp contrast with the $I V-I I I$ geometry. Consider a stack of three D7-branes along $z=0$ that has transverse intersection with a stack of two D7-branes along $t=0$. This is a type IIB configuration with $\mathrm{SU}(3) \times \mathrm{SU}(2)$ gauge symmetry; the strings localized at $z=t=0$ transform in the bifundamental representation $(\mathbf{3}, \mathbf{2})$, which can become quark doublets $(\mathbf{3}, \mathbf{2})_{1 / 6}$ after turning on worldvolume fluxes and engineering an embedding of the weak hypercharge. This type IIB configuration is equivalent to the an F-theory geometry that realizes an $I_{3}$ fiber along $z=0$ and an $I_{2}$ fiber along $t=0$, in which case

$$
\Delta=z^{3} t^{2} \tilde{\Delta}
$$

where $\tilde{\Delta}$ is a residual piece of the discriminant that is trivially computed in examples with a toric base.

The matter content of the theory can be determined by resolving the geometry or by studying a deformation of the geometry near the intersection of the branes. A first step in the case of a full deformation analysis is to study properties of the deformed geometry in an elliptic surface near the collision; this is the analysis we will perform. To do this, we choose an appropriate cross-section of the branes, as depicted for example by the dotted 
line on the left hand side of figure 1. A deformation of the geometry can yield the picture

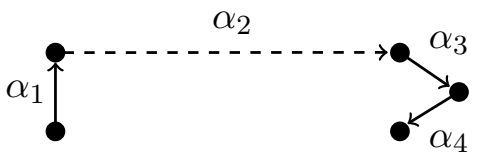

SU(2) Simple Roots SU(3) Simple Roots

from Deformed $I_{2} \quad$ from Deformed $I_{3}$

where the page describes the complex plane along the mentioned dotted line, and we note that the branes of the $\mathrm{SU}(2)$ and $\mathrm{SU}(3)$ gauge theories are now split.

Specifically, both gauge factors have been spontaneously broken, which allows us to study the now-massive states in the deformed geometry. Above, the arrows are string states that can be represented as vectors in $\mathbb{Z}^{5}$, specifically

$$
\alpha_{i}=e_{i}-e_{i+1}
$$

for an orthonormal basis $e_{i} \in \mathbb{Z}^{5}$. The set of states $\left\{\alpha_{1}, \alpha_{2}, \alpha_{3}, \alpha_{4}\right\}$ are simple roots of $\mathrm{SU}(5)$ and thus generate an entire adjoint of $\mathrm{SU}(5)$.

In the undeformed geometry all of these states become massless at $z=t=0$ since the branes collide; only those states in the adjoint of SU(5) that are in the adjoint of $\mathrm{SU}(2)$ are massless along the entire $t=0$ locus, and similarly for $\mathrm{SU}(3)$ adjoint states along $z=0$. Thus, the states that become massless at only $z=t=0$ are the ones that have one end on each of the different stacks of branes; these are roots of $\mathrm{SU}(5)$ that have a contribution from $\alpha_{2}$, the simple root represented by the dotted line above. These string states are

$$
\begin{gathered}
\alpha_{2}, \alpha_{2}+\alpha_{3}, \alpha_{2}+\alpha_{3}+\alpha_{4} \\
\alpha_{1}+\alpha_{2}, \alpha_{1}+\alpha_{2}+\alpha_{3}, \alpha_{1}+\alpha_{2}+\alpha_{3}+\alpha_{4}
\end{gathered}
$$

together with their negatives, and they fill out the (reducible) representation $(3, \overline{2}) \oplus(\overline{3}, 2)$ of $\mathrm{SU}(3) \times \mathrm{SU}(2)$, where the difference between $\overline{2}$ and 2 is the sign of the arrow into the $\mathrm{SU}(2)$ stack. This result matches the branching rule of an $\mathrm{SU}(5)$ adjoint

$$
24 \longrightarrow(8,1) \oplus(1,3) \oplus(3, \overline{2}) \oplus(\overline{3}, 2) \oplus(1,1)
$$

into representations of $\mathrm{SU}(3) \times \mathrm{SU}(2)$, in accord with the Katz-Vafa procedure. In summary, a $(3, \overline{2}) \oplus(\overline{3}, 2)$ of $\mathrm{SU}(3) \times \mathrm{SU}(2)$ is localized at $z=t=0$, which can in principle become a chiral supermultiplet of left-handed quark doublets after turning on flux and engineering an embedding of weak hypercharge.

There are a few particular features we would like to note about this well-known example.

- The only branes at the $\mathrm{SU}(3) \times \mathrm{SU}(2)$ intersection are the $\mathrm{SU}(3)$ and $\mathrm{SU}(2)$ brane stacks themselves.

- The states that become massless at the intersection are charged under both $\mathrm{SU}(3)$ and $\mathrm{SU}(2)$. 

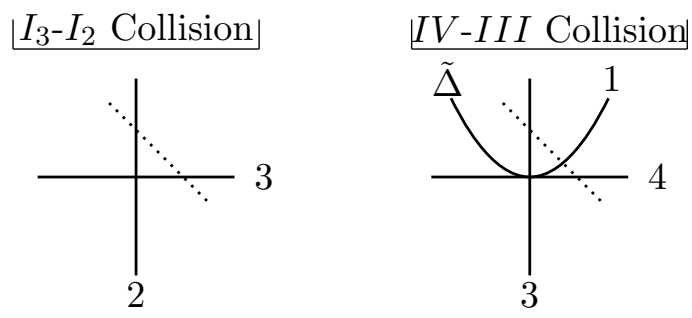

Figure 1. Displayed are the local geometries of an $I_{3}-I_{2}$ collision and a $I V-I I I$ collision, respectively. In both cases the $\mathrm{SU}(2)$ gauge theory is on the vertical line, the $\mathrm{SU}(3)$ gauge theory is on the horizontal line, and quark doublets are localized at the intersection of the solid lines; the integers denote the number of branes in each stack. Note the additional brane $\tilde{\Delta}$ participating in the intersection in the $I V-I I I$ case.

- Obtaining this geometry requires specializing in moduli space. Obtaining additional states — such as the other standard model fields - may require an additional specialization in moduli space.

We will see the first two of these statements are not true of the $I V$-III geometry. Furthermore, the third is not true when the $I V$-III collision is non-Higgsable.

Standard model matter representations from a $\boldsymbol{I V}-\boldsymbol{I I I}$ collision. We now turn to study the $I V-I I I$ geometry. As mentioned above, the main point of this section is that if one sets out to obtain quark doublets via colliding a type $I I I$ fiber with a type $I V$ fiber, one obtains not only these states, but also $\mathrm{SU}(3) \times \mathrm{SU}(2)$ representations that may realize the other standard model matter fields. Moreover, these states are all localized on the same matter curve in the threefold base.

Consider an F-theory model that realizes $\mathrm{SU}(3) \times \mathrm{SU}(2)$ gauge symmetry with a type $I V$ and type $I I I$ fiber, respectively, rather than the usual $I_{3}$ and $I_{2}$ of the type $I I B$ string. A Weierstrass model that realizes this possibility must be specified by the data

$$
f=z t^{2} \tilde{f} \quad g=z^{2} t^{2} \tilde{g}
$$

where $z, t, \tilde{f}$ and $\tilde{g}$ are (local) sections of $\mathcal{O}(Z), \mathcal{O}(T), \mathcal{O}\left(-4 K_{B}-Z-2 T\right)$, and $\mathcal{O}\left(-6 K_{B}-\right.$ $2(Z+T))$, respectively. The discriminant takes the form

$$
\Delta=z^{3} t^{4}\left(4 t^{2} \tilde{f}^{3}+27 z \tilde{g}^{2}\right) \equiv z^{3} t^{4} \tilde{\Delta} .
$$

where the residual discriminant $\tilde{\Delta}$ is a section of $\mathcal{O}\left(-12 K_{B}-3 Z-4 T\right)$. Assuming there is no outer monodromy on the type $I V$ fiber, which holds if $g=t^{2} g_{2}+\mathcal{O}\left(t^{3}\right)$ has $g_{2}$ a perfect square, then the seven-branes along $t=0$ and $z=0$ have gauge symmetry SU(3) and $\mathrm{SU}(2)$, respectively. We assume that the codimension two locus $z=t=0$ exists in the geometry, since this is is necessary to have the collision, and therefore quark doublets; in section 5 we present explicit examples realizing this possibility.

As in the case of the $I_{3}-I_{2}$ intersection, the seven-branes carrying $\mathrm{SU}(3)$ and $\mathrm{SU}(2)$ gauge theories intersect at $z=t=0$, where one expects the localization of massless charged 
states. There are two important differences, however. First, the SU(3) and SU(2) sevenbranes are stacks of four and three $(p, q)$ seven-branes, rather than stacks of three and two D7-branes; thus, the $\mathrm{SU}(3)$ and $\mathrm{SU}(2)$ stacks contribute more branes to the $z=t=0$ locus in a $I V-I I I$ collision than in an $I_{3}-I_{2}$ collision. Second, in the case of a $I V-I I I$ collision it is easy to see from the $z, t$ dependence of $\tilde{\Delta}$ that $\tilde{\Delta}=0$ always intersects $z=t=0$, unlike in an $I_{3}-I_{2}$ collision; i.e., there is always an additional brane, henceforth called the extra brane, and we will see that charged states may end on it. This corresponds to the observation mentioned earlier that the intersection of loci with orders of vanishing of $f, g, \Delta$ of $(1,2,3)$ and $(2,2,4)$ must have vanishing orders $(3,4,8)$, not $(3,4,7)$. This enhancement of the order of vanishing of $\Delta$ comes from an additional $I_{1}$ codimension one singularity that must also meet the $I V$-III intersection point. Thus, this intersection point might more properly be referred to as a $I V-I I I\left(-I_{1}\right)$ collision, with the parentheses indicating that the third brane is automatically produced when the first two singularities collide. The structure of the two intersecting geometries near the codimension two intersection is given in figure 1 .

The vanishing degrees $(3,4,8)$ of $f, g, \Delta$ at the intersection point corresponds to a Kodaira type $I V^{*}$ singularity, associated with an $E_{6}$ Dynkin diagram. We thus expect that the matter fields at the singularity should be those associated with a decomposition of the adjoint of $E_{6}$ when $\mathrm{SU}(3) \times \mathrm{SU}(2)$ is embedded as a subgroup. The deformation analysis using junctions provides a simple way of seeing explicitly how this decomposition works.

Let us therefore now deform the geometry in order to study the $\mathrm{SU}(3) \times \mathrm{SU}(2)$ representations of the states localized at the $I V-I I I\left(-I_{1}\right)$ collision. Consider a compact elliptically fibered Calabi-Yau variety in the special Weierstrass form (4.13) in a patch that gives a local model near the $I V-I I I$ intersection. Consider the deformation of the local model defined by

$$
(f, g) \longrightarrow(f, g+\epsilon)
$$

where $\epsilon$ may be the restriction of a deformation of the global model, depending on the example; alternatively it may simply be considered as a deformation of the local model. This deforms the discriminant to

$$
\Delta=4 t^{6} \tilde{f}^{3} z^{3}+27\left(t^{2} z^{2} \tilde{g}+\epsilon\right)^{2}
$$

and we see that the three and four seven-branes along $z=0$ and $t=0$, respectively, have split up; the $z=0$ and $t=0$ singular loci have been smoothed.

Given this deformation, we would like to study an elliptic surface nearby the $I V-I I I(-$ $I_{1}$ ) collision by taking a transverse slice, as displayed in figure 1 . Taking $\tilde{f}=\tilde{g}=1$ and defining the local coordinate

$$
p_{\perp}=t-\epsilon_{z} z-t_{0}
$$

where $\epsilon_{z} \in \mathbb{C}$ is a rotation factor and $t_{0}$ is an offset, we can define a family of transverse slices of the local geometry by $p_{\perp}=0$ for various values of the parameters. A generic pair $\left(t_{0}, \epsilon_{z}\right)$ should suffice; we take $\left(5,2 e^{i \pi / 5}\right)$ and $\epsilon=10$, in which case the deformed 
seven-branes intersect the transverse plane as
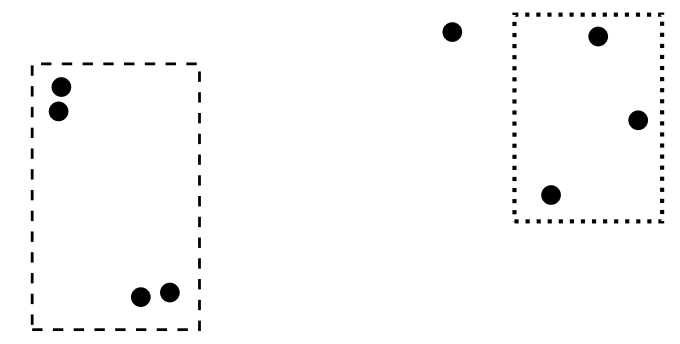

where the dashed box contains the split $\mathrm{SU}(3)$ branes of the type $I V$ fiber, the dotted box contains the split $\mathrm{SU}(2)$ branes of the type $I I I$ fiber, and the seven-brane on its own is the deformed extra brane. Using the techniques of $[27,28]$ to read off the vanishing one-cycles in the elliptic fiber above these marked points, beginning with the extra brane and moving clockwise, we find

$$
Z_{I V-I I I}=\left\{\pi_{2}, \pi_{1}, \pi_{3}, \pi_{2}, \pi_{1}, \pi_{3}, \pi_{1}, \pi_{3}\right\}
$$

which has associated I-matrix

$$
I=(\cdot, \cdot)=\left(\begin{array}{cccccccc}
-1 & \frac{1}{2} & -\frac{1}{2} & 0 & \frac{1}{2} & -\frac{1}{2} & \frac{1}{2} & -\frac{1}{2} \\
\frac{1}{2} & -1 & \frac{1}{2} & -\frac{1}{2} & 0 & \frac{1}{2} & 0 & \frac{1}{2} \\
-\frac{1}{2} & \frac{1}{2} & -1 & \frac{1}{2} & -\frac{1}{2} & 0 & -\frac{1}{2} & 0 \\
0 & -\frac{1}{2} & \frac{1}{2} & -1 & \frac{1}{2} & -\frac{1}{2} & \frac{1}{2} & -\frac{1}{2} \\
\frac{1}{2} & 0 & -\frac{1}{2} & \frac{1}{2} & -1 & \frac{1}{2} & 0 & \frac{1}{2} \\
-\frac{1}{2} & \frac{1}{2} & 0 & -\frac{1}{2} & \frac{1}{2} & -1 & -\frac{1}{2} & 0 \\
\frac{1}{2} & 0 & -\frac{1}{2} & \frac{1}{2} & 0 & -\frac{1}{2} & -1 & \frac{1}{2} \\
-\frac{1}{2} & \frac{1}{2} & 0 & -\frac{1}{2} & \frac{1}{2} & 0 & \frac{1}{2} & -1
\end{array}\right) .
$$

This is all of the data necessary to perform a first analysis of the string junctions near the collision.

Let us study the roots realized locally in the geometry by computing $\left\{J \in \mathbb{Z}^{8} \mid(J, J)=-2\right.$ and $\left.a(J)=0\right\}$. We find that it is composed of 72 junctions that fill out (together with the six Cartan elements $(0,0,0,0,0,0,0,0))$ an adjoint of $E_{6}$. A set of simple roots is given by

$$
\begin{aligned}
& \alpha_{1}=(0,0,0,0,1,0,-1,0) \\
& \alpha_{2}=(0,0,0,0,0,1,0,-1) \\
& \alpha_{3}=(0,0,0,-1,-1,-1,0,0) \\
& \alpha_{4}=(0,1,1,1,0,0,0,0) \\
& \alpha_{5}=(0,-1,0,1,1,0,1,1) \\
& \alpha_{6}=(-1,-1,-1,0,0,0,0,0)
\end{aligned}
$$

Note that $\alpha_{1}$ and $\alpha_{2}$ are string junctions that end only on the last four seven-branes; these are the simple roots of the SU(3) gauge theory. Similarly, $\alpha_{4}$ ends only on the second, 
third, and fourth, seven-brane; these are the simple roots of the $\mathrm{SU}(2)$ gauge theory. The only simple root that ends on the extra brane is $\alpha_{6}$.

This is an entire adjoint of $E_{6}$ in an elliptic surface near the $I V-I I I\left(-I_{1}\right)$ collision, and accordingly those junctions in the adjoint that aren't roots of $\mathrm{SU}(3)$ or $\mathrm{SU}(2)$ may give rise to matter multiplets. Since determining the $\mathrm{SU}(3) \times \mathrm{SU}(2)$ content of these states ultimately amounts to branching the adjoint of $E_{6}$, we must determine how $\mathrm{SU}(3)$ and $\mathrm{SU}(2)$ embed into $E_{6}$. To do this, we compute

$$
-C_{i j}=\left(\alpha_{i}, \alpha_{j}\right)=\left(\begin{array}{cccccc}
-2 & 1 & 0 & 0 & 0 & 0 \\
1 & -2 & 1 & 0 & 0 & 0 \\
0 & 1 & -2 & 1 & 1 & 0 \\
0 & 0 & 1 & -2 & 0 & 1 \\
0 & 0 & 1 & 0 & -2 & 0 \\
0 & 0 & 0 & 1 & 0 & -2
\end{array}\right)
$$

and find that it is the negative Cartan matrix. From this we construct the associated Dynkin diagram in figure 2, crossing out any node not associated with the $\mathrm{SU}(3) \times \mathrm{SU}(2)$ gauge symmetry.

The complete branching to $\mathrm{SU}(3) \times \mathrm{SU}(2)$ of this adjoint of $E_{6}$ is straightforward to compute. A detailed list of the states in resulting decomposition is given in table 5 . Besides the junctions in the adjoints of $\mathrm{SU}(3)$ and $\mathrm{SU}(2)$, there are junctions that transform in representations $(\mathbf{3}, \mathbf{2}),(\mathbf{3}, \mathbf{1}),(\mathbf{1}, \mathbf{2})$, and $(\mathbf{1}, \mathbf{1})$ (as well as, the usual conjugates of the first two representations where $\mathbf{3}$ is replaced by $\overline{\mathbf{3}}$ ). The junctions that end on the extra brane thus fill out precisely the set of $\mathrm{SU}(3) \times \mathrm{SU}(2)$ representations that are needed for the standard model matter fields, including the singlet representation needed for the righthanded lepton sector.

This is not a full analysis, and is not expected to reproduce the multiplicities of the matter fields arising at the intersection; for a given intersection of this form the deformation analysis could be extended to include a continuous family of surfaces in the vicinity of the singularity, and monodromy around one or the other of the branes can in general lead to an identification between sets of states that would reduce the multiplicity [27]. Indeed, we expect such a reduction in this case, since the charged spectrum with multiplicities computed in the $6 \mathrm{D}$ case in the following section shows only one field in each of the distinct representations under $\mathrm{SU}(3) \times \mathrm{SU}(2)$. One important lesson that we take from this deformation analysis, however, is that in addition to the charged fields there are also fields that transform in the singlet representation at the intersection point.

There is a simple way to understand why junctions with the $\mathrm{SU}(3) \times \mathrm{SU}(2)$ quantum numbers of the standard model fermions have appeared in this analysis. Notice that while we have a full adjoint of $E_{6}$ in the elliptic surface near the $I V-I I I\left(-I_{1}\right)$ collision, we have $\mathrm{SU}(3) \times \mathrm{SU}(2)$ gauge symmetry, and one could study how the $E_{6}$ states branch under the intermediate $\mathrm{SU}(5)$ or $\mathrm{SO}(10)$ whose corresponding simple roots are

$$
\text { SU(5) Simple Roots: } \quad\left\{\alpha_{1}, \alpha_{2}, \alpha_{3}, \alpha_{4}\right\}
$$




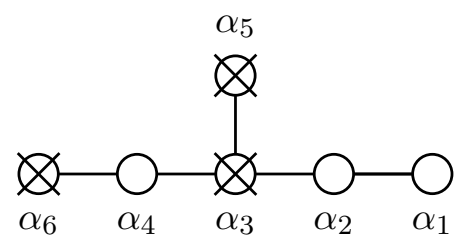

Figure 2. In an elliptic surface near a $I V-I I I\left(-I_{1}\right)$ collision the geometry exhibits an $E_{6}$ singularity, where the the SU(3) and SU(2) Dynkin diagram embed as displayed above. The crossed nodes are simple roots of $E_{6}$ whose associated states only become massless in codimension two. The left-most node corresponds to the $E_{6}$ simple root junction charged under the extra brane.

$$
\mathrm{SO}(10) \text { Simple Roots: } \quad\left\{\alpha_{1}, \alpha_{2}, \alpha_{3}, \alpha_{4}, \alpha_{5}\right\} \text {. }
$$

Note that $\alpha_{6}$ is the simple root lost in the $E_{6} \rightarrow \mathrm{SO}(10)$ branching, and that this is the only simple root junction ending on the extra brane. Accordingly, the states in the $E_{6}$ adjoint that end on the extra brane are those that are not roots of $\mathrm{SO}(10)$, which therefore obey the branching rule

$$
72 \rightarrow 45+16+16^{\prime}+1
$$

and fit into a $16+16^{\prime}$ of $\mathrm{SO}(10)$. Indeed this can be checked, and that the further branching of those junctions to $\mathrm{SU}(3) \times \mathrm{SU}(2)$ respect the decomposition of the 16 spinor of $\mathrm{SO}(10)$ into standard model representations. Thus, the representation theory of $\mathrm{SO}(10)$ plays an important role in this geometry, even though the geometry does not exhibit an SO(10) GUT.

We summarize by restating the result of this analysis: the $\mathrm{SU}(3) \times \mathrm{SU}(2)$ matter representations at the $I V-I I I\left(-I_{1}\right)$ collision include fields in the representations

$$
\left(\square_{3}, \square_{2}\right), \square_{3}, \square_{2}, \mathbf{1} .
$$

While the slice-based junction analysis we performed in this section does not nail down the multiplicities of these representations, the $6 \mathrm{D}$ anomaly analysis in the following section shows that each of the non-trivial representations appears with multiplicity one at the intersection point. The anomaly analysis, however, is not sensitive to the singlet representations identified by the junction analysis. The representations in (4.25) are precisely the $\mathrm{SU}(3) \times \mathrm{SU}(2)$ representations of the various matter fields in the standard model; thus, as we discussed further below, in models with appropriately engineered $G$-flux and weak hypercharge, it is possible that geometries of this type might realize all of the standard model fermions along the same matter curve.

\section{$5 \quad$ Examples}

In this section we provide some specific examples of F-theory compactifications giving type $I V$ and type $I I I$ singularities to show how some of the ideas of this paper are realized in concrete situations. We begin in section 5.1 with some simple examples in six-dimensional theories. In section 5.2 we give two examples of $4 \mathrm{D}$ F-theory models with non-Higgsable $\mathrm{SU}(3)$ gauge groups from Kodaira type $I V$ singularities, and two further examples that have 
non-Higgsable SU(3) gauge groups and Higgsable SU(2) gauge group factors. In section 5.3, we give an example of a $4 \mathrm{D}$ F-theory model that has a non-Higgsable $\mathrm{SU}(3) \times \mathrm{SU}(2)$ gauge group from intersecting type $I V$ and type $I I I$ singularities and an $\mathrm{SU}(3) \times \mathrm{SU}(2)$ example of type $I V-I V m$. These examples illustrate the structures involved in the different geometric realizations of the gauge group factors, and the different approaches that can be used to identify non-Higgsable structure in given compactification geometries.

\subsection{Warm-up: some 6D examples}

We begin with a few simple examples of F-theory compactifications to six dimensions with type $I I I$ and type $I V$ singularities. In six dimensions, as discussed earlier, the matter spectrum of an F-theory compactification is highly constrained, and in many situations uniquely determined, by the cancellation of gauge, gravitational, and mixed gauge-gravitational anomalies, and there are no complications such as G-flux that modify the spectrum beyond that determined by the geometry of the F-theory compactification.

In the simplest of these examples, where there are no non-Higgsable gauge group factors, we take the F-theory base manifold to be $B_{2}=\mathbb{P}^{2}$, and tune all codimension one singularities on linear $\mathbb{P}^{1}$ 's (complex lines) associated with the vanishing of coordinates $z, u$ (in a homogeneous coordinate system on $\mathbb{P}^{2}[s: u: z]$ ). Anomaly cancellation uniquely determines the matter spectrum of an $\mathrm{SU}(2)$ or $\mathrm{SU}(3)$ gauge group on such a curve, which has self-intersection +1 : for $\mathrm{SU}(2)$ there are 22 matter hypermultiplets transforming in the fundamental representation, and for $\mathrm{SU}(3)$ there are 24 fundamental hypers. (See, for example, [37], §2.5; the fact that only matter hypermultiplets in the fundamental representations can arise can be proven simply from the fact that matter representations of $\mathrm{SU}(n)$ associated with Young diagrams with more than one column can only be present when the $\mathrm{SU}(n)$ is realized on a curve of genus $g>0$ [75].)

We also consider six-dimensional compactifications with base surfaces that give rise to non-Higgsable $\mathrm{SU}(3)$ factors; such factors always arise on -3 curves in the base, as in the Hirzebruch surface $\mathbb{F}_{3}$, and in six dimensions there cannot be any matter charged under the resulting $\mathrm{SU}(3)$. In six dimensions, there are no non-Higgsable realizations of $\mathrm{SU}(2)$ that are relevant for the gauge groups of interest here; the only way that a non-Higgsable $\mathrm{SU}(2)$ can arise is in combination with either a $G_{2}$ or $\mathrm{SO}(7)$ gauge group factor [9].

\subsection{1 $\mathrm{SU}(2)$ in $6 \mathrm{D}$ models}

First, we illustrate the difference between tuning an $\mathrm{SU}(2)$ on the divisor $Z=\{z=0\}$ on $B_{2}=\mathbb{P}^{2}$ with a type $I I I$ singularity versus a type $I_{2}$ singularity. In local coordinates $z, u$, we can expand

$$
\begin{aligned}
& f(z, u)=f_{0}^{(12)}(u)+f_{1}^{(11)}(u) z+\cdots+f_{12}^{(0)} z^{12} \\
& g(z, u)=g_{0}^{(18)}(u)+g_{1}^{(17)}(u) z+\cdots+g_{18}^{(0)} z^{18}
\end{aligned}
$$

where $f, g$ are polynomials in $z, u$ of degrees 12,18 respectively, since the anti-canonical class of $\mathbb{P}^{2}$ is $-K=3 H$, with $H$ the hyperplane class, and $f, g$ are sections of $\mathcal{O}(-4 K)$ and $\mathcal{O}(-6 K)$, respectively. To tune an $\mathrm{SU}(2)$ on $Z$ with a type $I_{2}$ singularity, we need 
to arrange for $\Delta=4 f^{3}+27 g^{2}$ to vanish to order $z^{2}$. We choose $f_{0}^{(12)}=-3 \phi_{(6)}^{2}, g_{0}^{(18)}=$ $2 \phi_{(6)}^{3}, g_{1}^{(17)}=-\phi_{(6)} f_{1}^{(11)}$, and find

$$
\Delta=9 z^{2} \phi_{(6)}^{2}\left(-\left(f_{1}^{(11)}\right)^{2}+12 \phi_{(6)} g_{2}^{(16)}+12 \phi_{(6)}^{2} f_{2}^{(10)}\right)+\mathcal{O}\left(z^{3}\right)
$$

As discussed, for example, in [31], the 22 points where the term in parentheses vanishes are locations where the residual discriminant locus intersects $Z$, the singularity is enhanced to $I_{3}$, and there is a fundamental matter representation of the $\mathrm{SU}(2)$ through the standard Katz-Vafa rank one enhancement [29]. At the points where $\phi_{(6)}$ vanishes, there would be an antisymmetric representation if the gauge group were $\mathrm{SU}(n)$ with $n>3$ (or an anti-fundamental for $n=3$ ), but this representation is trivial for $n=2$.

Now, let us consider the tuning of a type $I I I$ singularity on the same locus $Z$. To achieve this we set $f_{0}^{(12)}=g_{0}^{(18)}=g_{1}^{(17)}=0$. The discriminant is then

$$
\Delta=4\left(f_{1}^{(11)}\right)^{3} z^{2}+\mathcal{O}\left(z^{3}\right)
$$

We see that at points where $f_{1}^{(11)}$ vanishes, the type $I I I$ singularity is enhanced to a type $I V(2,2,4)$ singularity. Since anomaly cancellation uniquely determines the matter content, which must consist of 22 matter fields in the fundamental representation of SU(2), we see that at each such enhancement point there are two fundamental matter fields. This multiplicity can also be determined by an analysis of the singularity structure, as described in $[30,33]$. This shows that when a codimension one type III singularity is enhanced at a codimension two locus to a type $I V$ singularity, there is fundamental matter, with multiplicity two in the case of six-dimensional theories.

\subsection{2 $\mathrm{SU}(3)$ in six dimensions}

A similar argument to the one above shows that while tuning an $\mathrm{SU}(3)$ on an $I_{3}$ gives 24 fundamental representations at separate points on a codimension one curve (really 21 fundamentals where there is an enhancement $I_{3} \rightarrow I_{4}$, and 3 "antisymmetrics" where $\phi_{(6)}=\varphi_{(3)}^{2}$ vanishes), when the $\mathrm{SU}(3)$ is tuned on a type $I V$ singularity, we have $f_{0}^{(12)}=$ $f_{1}^{(11)}=g_{0}^{(18)}=g_{1}^{(17)}=0$, and $g_{2}^{(16)}=\left(\gamma^{(8)}\right)^{2}$, where the condition that $g_{2}$ is a perfect square is necessary for the monodromy at the type $I V$ locus to give an $\mathrm{SU}(3)$ instead of an $\mathrm{SU}(2)$ gauge group factor $[20,21]$. In this situation,

$$
\Delta=27\left(\gamma^{(8)}\right)^{4} z^{4}+\mathcal{O}\left(z^{5}\right)
$$

and the 24 matter fields arise at eight places where the type $I V$ singularity is enhanced to a $I_{0}^{*}(2,3,6)$ singularity. Thus, at codimension two loci where a type $I V$ singularity giving a $\mathrm{SU}(3)$ is enhanced to a type $I_{0}^{*}$ singularity, there is matter in the fundamental representation of $\mathrm{SU}(3)$, with multiplicity 3 in the case of six-dimensional theories. This result was also derived in [33]. 


\subsection{3 $\mathrm{SU}(3) \times \mathrm{SU}(2)$ in six dimensions}

Now, let us consider a situation where type $I I I$ and type $I V$ singularities intersect transversely with an $\mathrm{SU}(3)$ on the type $I V$. On $\mathbb{P}^{2}$ this can be arranged by choosing

$$
\begin{aligned}
& f=z u^{2} \tilde{f} \\
& g=z^{2} u^{2}\left(\gamma_{(7)}\right)^{2}+\cdots
\end{aligned}
$$

which gives

$$
\Delta=z^{3} u^{4}\left(27 z \gamma^{4}+4 u^{2} \tilde{f}^{3} \cdots\right)
$$

so the singularity at the intersection point $z=u=0$ is type $I V^{*}(3,4,8)$. Since $\tilde{f}, \gamma$ have degrees 9,7 respectively, we see that the type $I I I$ curve $Z$ is enhanced to type $I V$ in the fashion described above at 9 points away from the $I V-I I I$ intersection, giving 18 fundamentals, and the type $I V$ curve $U$ is enhanced to type $I_{0}^{*}$ at 7 points away from the intersection, giving 21 fundamentals. Anomaly cancellation indicates that there is one bifundamental $(\mathbf{3}, \mathbf{2})$ at the $I V$ - $I I I$ intersection point, and requires a total of $22 \square_{2}^{\prime} s$ and $24 \square_{3}{ }^{\prime} s$. Thus, anomaly cancellation requires that there is one $\operatorname{SU}(2)$ fundamental $(\mathbf{1}, \mathbf{2})$ and one $\mathrm{SU}(3)$ fundamental $(\mathbf{3}, \mathbf{1})$ that must also be localized at the intersection point.

To summarize, in the $6 \mathrm{D}$ model the $\mathrm{SU}(3) \times \mathrm{SU}(2)$ charged matter localized at the $I V$ - III intersection consists of one hypermultiplet each in the $(\mathbf{3}, \mathbf{2}),(\mathbf{1}, \mathbf{2}),(\mathbf{3}, \mathbf{1})$ representations. These non-trivial $\mathrm{SU}(3) \times \mathrm{SU}(2)$ representations match those of the deformation analysis of the previous section. Note, as mentioned earlier, that this anomaly analysis is not sensitive to uncharged $\mathrm{SU}(3) \times \mathrm{SU}(2)$ singlets that are localized at the $I V-I I I\left(-I_{2}\right)$ collision, which the deformation analysis suggests must exist.

We can use a similar analysis to construct an $\mathrm{SU}(3) \times \mathrm{SU}(2)$ gauge group through an intersection of type $I V$ and $I_{2}$ singularities, beginning with the form of the discriminant (5.1) given the $I_{2}$ locus on $z=0$, and where $u\left|\phi, u^{2}\right| f_{1}^{(11)}, \ldots$. The result is basically the same as in the preceding example, except that 19 fundamentals arise on the $I_{2}$ locus away from the $I V-I_{2}$, occurring at 19 distinct points each containing a single multiplet, unlike the type $I I I \mathrm{SU}(2)$ case where the multiplets appear in pairs. Localized at the $6 \mathrm{D} I V-I_{2}$ intersection there is one bifundamental $(\mathbf{3}, \mathbf{2})$, and one fundamental $(\mathbf{3}, \mathbf{1})$, but no $(\mathbf{1}, \mathbf{2})$.

Finally, we consider the $I V-I V m$ intersection. To understand the spectrum here, it is simplest to begin with a $I V-I V$ intersection where both divisors carry an $\mathrm{SU}(3)$. In this case, we have $f=z^{2} u^{2} \tilde{f}, g=z^{2} u^{2}\left(\gamma_{(7)}\right)^{2}+\cdots$. Just as for the $\mathrm{SU}(3)$ of the $I V-I I I$ intersection, each of the SU(3)'s has 21 fundamentals localized at 7 points away from the intersection, and there is a $(\mathbf{3}, \mathbf{3})$ localized at the intersection, which suffices to saturate the anomalies. We can now break one of the SU(3)'s by Higgsing two of the fundamentals. Geometrically, this can be done in a way that corresponds to turning on a nontrivial monodromy for one of the $\mathrm{SU}(3)$ factors; this occurs if we take $g=z^{2} u^{2}\left(\gamma_{(7)}^{2}+u \gamma_{13}\right)+\mathcal{O}\left(z^{3}\right)$. The resulting $\mathrm{SU}(3) \times \mathrm{SU}(2)$ has $21(\mathbf{3}, \mathbf{1})$ 's localized away from the intersection, $19(\mathbf{1}, \mathbf{2})$ 's localized away from the intersection, and one each of $(\mathbf{3}, \mathbf{2}),(\mathbf{3}, \mathbf{1})$ localized at the intersection.

The detailed counting from the geometry here is rather subtle; following [33, 76] the $\mathrm{SU}(2)$ fundamentals are nonlocal matter on the monodromy cover of the SU(2) curve. In 
particular, there are 14 branch points in the monodromy cover. The total space is thus a 14 times branched cover of a rational curve, which has genus 6 . Each branch point contributes a half hypermultiplet in the fundamental of $\mathrm{SU}(2)$, and each contribution to the genus gives an additional 2 nonlocal fundamentals. This gives a total of $2 \times 6+7=19$ fundamentals of $\mathrm{SU}(2)$ that are either nonlocal or localized away from the intersection point, confirming the preceding Higgsing analysis, and showing that the matter at the intersection point is simply $(\mathbf{3}, \mathbf{2})+(\mathbf{3}, \mathbf{1})$.

\subsubsection{Non-Higgsable SU(3) in six dimensions}

In six dimensions, the complete set of non-Higgsable clusters was determined in [9]. A nonHiggsable $\mathrm{SU}(3)$ arises on a type $I V$ singularity over any curve in the (two-dimensional) base that has self-intersection -3 and that does not intersect any curve of self-intersection -2 or below. Anomaly cancellation in the $6 \mathrm{D}$ theory shows that any non-Higgsable SU(3), which must lie on a -3 curve, cannot have any associated charged matter fields. This can also be seen geometrically, as argued below. Since a non-Higgsable SU(3) cannot have any charged matter, it is not possible to have a non-Higgsable $\mathrm{SU}(3)$ intersecting a Higgsable $\mathrm{SU}(2)$. Unlike for $\mathrm{SU}(3)$, there are no non-Higgsable clusters in six dimensions that give rise to the gauge groups $\mathrm{SU}(2)$ or $\mathrm{SU}(3) \times \mathrm{SU}(2)$. The only cases in which a non-Higgsable $\mathrm{SU}(2)$ gauge group factor can arise are in non-Higgsable clusters that support gauge groups $G_{2} \times \mathrm{SU}(2)$ or $\mathrm{SU}(2) \times \mathrm{SO}(7) \times \mathrm{SU}(2)$. As we show in the next subsections 5.2, 5.3, while $\mathrm{SU}(3) \times \mathrm{SU}(2)$ thus cannot be realized in six dimensions in such a way that either or both of the factors are non-Higgsable, in four dimensions a non-Higgsable $\mathrm{SU}(3)$ can be combined either with a Higgsable or non-Higgsable SU(2).

The simplest explicit example of a non-Higgsable SU(3) in a six-dimensional F-theory model is on the base $\mathbb{F}_{3}$. This base has a -3 curve, over which $f, g$ must vanish to degrees 2,2 respectively, with only a single monomial in $g_{2}$, giving a type $I V$ singularity carrying a $\mathrm{SU}(3)$ gauge group. This result is well known from the early days of F-theory constructions with dual heterotic models [3], and can be obtained in a variety of ways, each of which generalizes to four dimensional constructions. The toric approach can be used, as for $\mathbb{F}_{8}$ in section 3.2.

A convenient and general way of determining non-Higgsable structures in the toric approach uses the language of toric fans [56]. For $\mathbb{F}_{m}$ the toric diagram consists of the set of rays $v_{1}=(0,1), v_{2}=(1,0), v_{3}=(0,-1), v_{4}=(-1,-m)$; the rays live in an integral lattice, $v_{i} \in N=\mathbb{Z}^{2}$, and each ray corresponds to an effective divisor in the surface, with $v_{3}$ corresponding to the curve of self-intersection -3 . The set of monomials in the Weierstrass model is given by the set of points in the dual lattice $m=(a, b) \in M=N^{*}$ that satisfy, for $f$ and $g$ respectively, $\left\langle m, v_{i}\right\rangle \geq-4,-6$ for all rays $v_{i}$. For a given $m$ in $f, g$, the degree of vanishing of $m$ on the curve $S$ of self-intersection -3 is given by $4+\left\langle m, v_{3}\right\rangle, 6+\left\langle m, v_{3}\right\rangle$ respectively. It is straightforward to check that there are no monomials in $f$ or $g$ of degrees 0 or 1 on $S$, and only one monomial in $g$ (associated with $m=(-4,4)$ ) of degree 2, confirming that there is a type $I V$ singularity giving a gauge group $\mathrm{SU}(3)$ over this curve. This approach is explained and applied systematically for toric F-theory base surfaces in [48]. 
More abstractly, using the language of algebraic geometry as described in [9], the anticanonical class of the base $\mathbb{F}_{3}$ is $-K=2 S+5 F$, where in toric language $F$ corresponds to the ray $v_{2}\left(\sim v_{4}\right)$, and the curves $S, F$ have the intersection properties $S \cdot S=-3, S \cdot F=$ $1, F \cdot F=0$. Since the irreducible curve $S$ has negative self-intersection, it is rigid. Since $-4 K \cdot S=-4$, the divisor $-4 K$ must contain 2 copies of $S$, which means that $f$, as a section of the line bundle $\mathcal{O}(-4 K)$ associated with $-4 K$, must vanish to degree 2 over $S$. Similarly, $g$ must also vanish on $S$ to degree 2, giving again the type $I V$ singularity. We use variations on each of these approaches in identifying non-Higgsable clusters in the following $4 \mathrm{D}$ examples. Finally, as mentioned above, the F-theory model on $\mathbb{F}_{3}$ has a dual heterotic description [2] in terms of a compactification of heterotic $E_{8} \times E_{8}$ theory on a K3 surface, where the total instanton number is divided between the two gauge factors as $24=15+9$. On the side with 9 instantons, the generic bundle has $E_{6}$ structure, so the resulting gauge group is a non-Higgsable SU(3) gauge group with no charged matter. Thus, non-Higgsable clusters in cases with no matter are familiar structures also from the heterotic point of view.

Note that the absence of matter for any $6 \mathrm{D}$ non-Higgsable $\mathrm{SU}(3)$ can be proven generally from the algebraic geometry point of view. As shown in [9], a non-Higgsable SU(3) can only arise on a rational curve $C$ of self-intersection $C \cdot C=-3$. Since $-K \cdot C=-1$, $-n K$ must contain $C$ as an irreducible component $\lceil n / 3\rceil$ times; this is quantified by the Zariski decomposition over the rationals $-K=C / 3+X$, where the coefficient of $C$ is determined by the condition that it is the minimal value possible such that the residual component $X$ is effective and satisfies $X \cdot C \geq 0$. We then have $-6 K=2 C+X_{6}$, where the residual component $X_{6}$ satisfies $X_{6} \cdot C=0$, and $-12 K=4 C+X_{12}, X_{12} \cdot C=0$. The last condition shows that the discriminant locus can always be written as $\Delta=z^{4} \tilde{\Delta}$, where $\tilde{\Delta}$ has a vanishing locus that does not intersect $z=0$; therefore, there is no associated matter.

As we see below, the corresponding constraint is weaker in four dimensions, where a non-Higgsable SU(3) can have (geometric) charged matter, and can be realized in combination with a Higgsable or non-Higgsable intersecting $\mathrm{SU}(2)$ factor.

\subsection{D models with non-Higgsable SU(3) groups}

There are a wide range of $4 \mathrm{D}$ models with type $I V$ singularities on some divisor giving a non-Higgsable gauge group. The simplest case is an F-theory model on the base $B_{3}=$ $\mathbb{P}^{1} \times \mathbb{F}_{3}$. In this case the toric analysis is almost precisely identical to the $\mathbb{F}_{3}$ case described above. The $3 \mathrm{D}$ toric fan is generated by the rays

$$
\begin{aligned}
w_{i} & =\left(v_{i}, 0\right), i \in\{1, \ldots, 4\} \\
w_{5,6} & =(0,0, \pm 1)
\end{aligned}
$$

To specify a complete 3D toric fan, we must also specify which pairs of rays span $2 \mathrm{D}$ cones in the fan in a way that triangulates $S^{2}$; we do that in this case by having a $2 \mathrm{D}$ cone generated by $\left\{w_{5}, w_{i}\right\}$ and $\left\{w_{6}, w_{i}\right\}$ for each $i<5$, in addition to the $2 \mathrm{D}$ cones inherited from $\mathbb{F}_{3}$ associated with the pairs $\left\{w_{i}, w_{i+1}\right\},\left\{w_{4}, w_{1}\right\}$. The conditions on monomials are as before. In this case, however, $g_{2}$ in the expansion of $g$ around the divisor $D_{3}$ is a function of the extra coordinate with 13 independent parameters, so generically is not a perfect square, so 
this gives a non-Higgsable $\mathrm{SU}(2)$ gauge group. A more constrained construction is needed to get the correct monodromy for an $\mathrm{SU}(3)$ on a type $I V$ singularity in 4 dimensions.

A general class of $4 \mathrm{D}$ F-theory compactifications with heterotic duals were considered in [22], based on manifolds $B_{3}$ with the form of a $\mathbb{P}^{1}$ bundle over a base $B_{2}$. When the base $B_{2}$ is a generalized del Pezzo surface, there is a smooth heterotic dual on a Calabi-Yau threefold that is an elliptic fibration over $B_{2}$. When the base $B_{2}$ is toric, then there is a simple toric description of the threefold, with the rays

$$
\begin{aligned}
w_{i} & =\left(v_{i}, t_{i}\right), i=1, \ldots, N \\
w_{ \pm} & =(0,0, \pm 1) .
\end{aligned}
$$

Here, the $2 \mathrm{D}$ rays $v_{i}$ give a toric description of $B_{2}$, there are $2 \mathrm{D}$ cones connecting each of $w_{ \pm}$to each of the $w_{i}$, in addition to the full set of $2 \mathrm{D}$ cones inherited from $B_{2}$. The integer parameters $t_{i}$ represent a divisor $T=\sum_{i} t_{i} D_{i}$, which describes the "twist" of the $\mathbb{P}^{1}$ bundle; the divisors $D_{ \pm}$(which are the zero and infinity sections of the $\mathbb{P}^{1}$ bundle) corresponding to the rays $w_{ \pm}$are sections of the $\mathbb{P}^{1}$ bundle, with normal bundles within $B_{3}$ given by $\mp T$. Note that two of the parameters $t_{i}$ are redundant and can be set to 0 by a linear transformation of the basis of the lattice $N=\mathbb{Z}^{3}$ that leaves the third axis unchanged.

The roughly 4000 bases $B_{3}$ that are $\mathbb{P}^{1}$ bundles over a toric generalized del Pezzo $B_{2}$ were enumerated in [22], and the associated non-Higgsable gauge groups were determined. Of these, over 100 had a non-Higgsable $\mathrm{SU}(3)$ on a type $I V$ singularity, and no further non-Higgsable gauge group. Some simple examples are given by $B_{2}=\mathbb{F}_{0}=\mathbb{P}^{1} \times \mathbb{P}^{1}$ with a twist $T=3 S+3 F$, and $B_{2}=\mathbb{F}_{2}$ with a twist $T=3 S+6 F$. (These are the only cases of non-Higgsable $\mathrm{SU}(3)$ 's for the class of $\mathbb{P}^{1}$ bundles over Hirzebruch surfaces $\mathbb{F}_{m}$, sometimes denoted by $\mathbb{F}_{m q r}$ ). The non-Higgsable $\mathrm{SU}(3)$ in these cases can be verified by a direct computation using toric monomials. More abstractly, as described in [22], the coefficients of $f$ in an expansion $f=f_{0}+f_{1} z+\cdots$ around the divisor $D=D_{+}$at a local coordinate $z=0$ are sections of line bundles on $D$

$$
f_{k} \in \mathcal{O}\left(-4 K_{D}-(4-k) T\right),
$$

and similarly for expansions of $g$ and $\Delta$, with 4 replaced by 6 and 12 respectively. For the Hirzebruch surface $\mathbb{F}_{m},-K=2 S+(2+m) F$. Thus, for the $3 \mathrm{D}$ base defined as a $\mathbb{P}^{1}$ bundle over $\mathbb{F}_{0}$ with twist $T=3(S+F)$, the expansion around $D_{+}$has $f_{k} \in \mathcal{O}((3 k-4)(S+F))$, and $g_{k} \in \mathcal{O}((3 k-6)(S+F))$. There can only be a section when the relevant divisor is effective, which occurs when the numerical coefficient $3 k-4$ or $3 k-6$ is nonnegative. This shows that $f, g$ vanish to degrees 2,2 at $D_{+}$. Furthermore, since $g_{2} \in \mathcal{O}(0)$ is a constant, it is a perfect square and the gauge group is $\mathrm{SU}(3)$. A similar argument gives a type $I V$ $\mathrm{SU}(3)$ singularity in the $\mathbb{F}_{2}$ case described above. Note that in both of these cases, $g_{2}$ and $\Delta_{4}$ are sections of the trivial line bundle. This means that the residual discriminant locus does not intersect the divisor carrying the $\mathrm{SU}(3)$ gauge group, so just as in the $6 \mathrm{D}$ cases described in the previous section, it is not possible to realize an $\mathrm{SU}(2)$ on a divisor intersecting the divisor carrying the type $I V$ singularity, even by tuning a Higgsable $\mathrm{SU}(2)$. Such a tuning would cause $g_{2}$ to vanish identically, pushing the original type $I V$ singularity to at least an $I_{0}^{*}$ singularity that carries a larger gauge group — at least $G_{2}$. 
There are, however, many other instances of non-Higgsable $\mathrm{SU}(3)$ gauge groups in the list of examples found in [22], which involve $\mathbb{P}^{1}$ bundles over bases that are more complicated, formed by blowing up Hirzebruch surfaces $\mathbb{F}_{m}$ at one or more points. In these more complicated constructions there can be curves that carry matter charged under the non-Higgsable $\mathrm{SU}(3)$, and in some cases additional divisors on which $\mathrm{SU}(2)$ factors can be tuned to intersect with the non-Higgsable SU(3) without increasing the singularity type of the original type $I V$ singularity locus. This gives a variety of situations in which we can have a non-Higgsable SU(3) combined with a Higgsable $\mathrm{SU}(2)$ in a $4 \mathrm{D}$ F-theory model. Two simple examples of this arise by blowing up points in the bases $\mathbb{F}_{0}, \mathbb{F}_{2}$ of the two non-Higgsable SU(3) examples described above.

First, consider blowing up $F_{0}=\mathbb{P}^{1} \times \mathbb{P}^{1}$ at a point, which gives the del Pezzo surface $d P_{2}$. This surface can be described torically, and we can construct a $3 \mathrm{D}$ base $B_{3}$ as a $\mathbb{P}^{1}$ bundle over this surface, with the toric rays $w_{i}=(0,1,0),(1,0,0),(1,-1,1)(0,-1,3),(-1,0,3)$. It is straightforward to check using explicit monomials that, like the preceding construction using $F_{0}$, this $4 \mathrm{D}$ F-theory model has a non-Higgsable $\mathrm{SU}(3)$ on the divisor $D_{+}$, and that furthermore a type III (Higgsable) $\mathrm{SU}(2)$ can be tuned on the divisor $D_{3}$ (corresponding to the exceptional divisor of the blowup on $F_{0}$ ), without enhancing the type $I V$ singularity on $D_{+}$. This can be seen more abstractly using the divisor structure of the base. The anti-canonical class of the new $2 \mathrm{D}$ base $d P_{2}$ can be written as $-K_{2}=2 S+2 F+3 E$, where $S, E, F$ correspond to the toric divisors $D_{2}, D_{3}, D_{4}$ in the preceding fan description. In this notation, the twist of the $\mathbb{P}^{1}$ bundle is $T=3 S+4 E+3 F$. (Note that, as above, there is a two-parameter family of equivalences on the twists, corresponding to the two equivalence relations on the divisors of a $2 \mathrm{D}$ toric fan - often referred to as the StanleyReisner ideal - which allows us to eliminate two of the divisors in the base $B_{2}$ in these expressions for $-K_{2}, T$.) In the expansion around $D_{+}$, the Weierstrass coefficient $g_{2}$ is now a section of the line bundle $\mathcal{O}(2 E)$. In terms of the Zariski decomposition, this means that $-6 K_{3}=2 D_{+}+X$, where $X$ can have a nontrivial intersection with $D_{+}$along the curve $D_{+} \cap E$. This means that the non-Higgsable $\mathrm{SU}(3)$ in this construction can have geometric charged matter, which is a necessary condition for the possibility of constructing a Higgsable $\mathrm{SU}(2)$ like the one that can be realized in this geometry.

A second example of a 4D theory with a non-Higgsable SU(3) and a Higgsable SU(2) can be found in a similar way by modifying the example with a non-Higgsable SU(3) constructed from a $\mathbb{P}^{1}$ bundle over $F_{2}$. Blowing up a generic point on the surface $F_{2}$ gives a generalized del Pezzo surface with a toric description. A toric $\mathbb{P}^{1}$ bundle over this surface can be constructed using the rays $w_{i}=(0,1,0),(1,0,0),(0,-1,3),(-1,-2,6),(-1,-1,4)$. Again, explicit checking of monomials demonstrates a non-Higgsable SU(3) on $D_{+}$, which can be combined with a Higgsable $\mathrm{SU}(2)$ on $D_{5}$. In the more abstract language, we have $-K=2 S+4 F+3 E$, where $S, F, E$ correspond to $D_{3}, D_{4}, D_{5}$ ( $E$ is the exceptional divisor in the blown up base surface $B_{2}$, as before), and $T=3 S+6 F+4 E$. Just as in the preceding case, $g_{2} \in \mathcal{O}(2 E)$, giving the possibility of charged matter under the $\mathrm{SU}(3)$, and matching with the existence of the Higgsable $\mathrm{SU}(2)$ on the divisor $E=D_{5}$.

A variety of further constructions can be realized in this way on base surfaces that involve further blowups; we have identified 27 configurations that allow for this possibility from the toric constructions enumerated in [22]. 


\section{$5.34 \mathrm{D}$ models with non-Higgsable $\mathrm{SU}(3) \times \mathrm{SU}(2)$}

Models with non-Higgsable product gauge groups such as $\mathrm{SU}(3) \times \mathrm{SU}(2)$ arising on intersecting divisors do not appear in the class of models considered in [22], since in F-theory models with smooth heterotic duals gauge groups can only arise on the sections $D_{ \pm}$of a $\mathbb{P}^{1}$ bundle. By considering a more general class of bases $B_{3}$ that are $\mathbb{P}^{1}$ bundles over the more general class of toric surfaces $B_{2}$ identified in [48] that can act as bases for elliptically fibered CalabiYau threefolds, including those bases that include curves of self-intersection -3 or below, we find models with more general non-Higgsable product group structures. A systematic scan over such more general F-theory $\mathbb{P}^{1}$ bundle bases (more general results from which will appear elsewhere [50]) reveals a number of models with non-Higgsable $\mathrm{SU}(3) \times \mathrm{SU}(2)$ gauge groups coming from intersecting $I V-I I I\left(-I_{1}\right)$ singularities. An example of such a model arises for a toric base constructed by blowing up the Hirzebruch surface $\mathbb{F}_{2}$ at two distinct points on the -2 curve $S$. A $\mathbb{P}^{1}$ bundle over this base can be constructed using the toric rays

$$
\begin{aligned}
& w_{1}=(0,1,0) \\
& w_{2}=(1,0,0) \\
& w_{3}=(1,-1,1) \\
& w_{4}=(0,-1,2) \\
& w_{5}=(-1,-3,6) \\
& w_{6}=(-1,-2,5) \\
& w_{ \pm}=(0,0, \pm 1),
\end{aligned}
$$

with cone structure as described in the previous subsection. A direct numerical computation using toric monomials confirms that this elliptically fibered Calabi-Yau fourfold has type $I V$ and type $I I I$ singularities on the divisors $D_{4}, D_{+}$, giving rise to a non-Higgsable gauge group $\mathrm{SU}(3) \times \mathrm{SU}(2)$. This can also be confirmed using a more abstract analysis on the surfaces and curves analogous to the method used above for analyzing the components of $f, g$ around a fixed divisor. A more general description of this approach will appear in [23]; in this case the analysis is a simple generalization of what is used above. For a local analysis around $D_{+}$, we have $-K_{+}=D_{3}+2 D_{4}+5 D_{5}+4 D_{6}$, with a twist (corresponding to the normal bundle of $D_{+}$) of $T_{+}=D_{3}+2 D_{4}+6 D_{5}+5 D_{6}$. So, for example, $g_{1}$ in an expansion around $D_{+}$is a section of the line bundle associated with the divisor $-6 K_{+}-5 T_{+}=$ $D_{3}+2 D_{4}-D_{6}$, which is not effective, so $g$ vanishes to degree at least 2 on $D_{+}$. Checking the other cases shows that we have a type $I I I$ singularity on $D_{+}$. The divisor $D_{4}$ can be seen from the local toric structure to have the geometry of a Hirzebruch surface $\mathbb{F}_{1}$, with anticanonical class $-K_{4}=2 D_{-}+3 D_{5}$, and normal bundle corresponding to a "twist" $T=4 D_{5}$. Analyzing the local structure of $f, g$ with this data gives a type $I V(2,2,4)$ singularity without monodromy, confirming the $\mathrm{SU}(3) \times \mathrm{SU}(2)$ non-Higgsable cluster in this geometry.

We conclude the set of examples with an example exhibiting a non-Higgsable $\mathrm{SU}(3) \times$ $\mathrm{SU}(2)$ of type $I V-I V m$, that is where the two gauge factors are realized on intersecting type $I V$ divisors, one without monodromy and one with. The base is found by blowing up $\mathbb{F}_{0}$ (or $\left.\mathbb{F}_{1}=d P_{1}\right)$ at a point giving a $d P_{2}$, and then blowing up at the two intersection points be- 
tween pairs of the three -1 curves in $d P_{2}$. The resulting base is toric, with a cyclic sequence of pairwise intersecting effective toric divisors of self-intersections $0,-2,-1,-3,-1,-2,0$, corresponding to the rays in the base geometry. With an appropriate twist we form a $\mathbb{P}^{1}$ bundle over this base with rays

$$
\begin{aligned}
& w_{1}=(0,1,0) \\
& w_{2}=(1,0,0) \\
& w_{3}=(2,-1,0) \\
& w_{4}=(1,-1,1) \\
& w_{5}=(1,-2,3) \\
& w_{6}=(0,-1,3) \\
& w_{7}=(-1,0,3) \\
& w_{ \pm}=(0,0, \pm 1) .
\end{aligned}
$$

Direct computation using toric monomials verifies that there are type $I V$ singularities on the divisors $D_{+}, D_{4}$, with monodromy in the latter case so the gauge group is $\mathrm{SU}(3) \times \mathrm{SU}(2)$.

\section{Directions for further development}

In this paper we have primarily focused on the geometric structure of non-Higgsable nonabelian gauge groups. We have identified the various ways in which the nonabelian part of the standard model gauge group $\mathrm{SU}(3) \times \mathrm{SU}(2)$ can arise in F-theory compactifications to four- and six-dimensional supergravity theories. To find a realistic way of realizing the full standard model in F-theory using a non-Higgsable SU(3), a number of further aspects of these scenarios must be analyzed in more detail. In particular, at least one $U(1)$ factor must be included in the gauge group, G-flux must be chosen so as not to alter the gauge group realized at the geometric level, and the details of the matter content (including multiplicities and chirality) must be worked out. In this section we give a brief discussion of the issues involved in these more detailed aspects of a complete F-theory description of the standard model of particle physics.

We would like to comment that although a careful analysis of $U(1)$ factors and chiral matter will be necessary to obtain a realistic model in the context of the non-Higgsable $\mathrm{SU}(3)$ gauge groups that we are considering here, recent works $[67,68]$ have given constructions of a chiral spectrum and weak hypercharge in non-GUT realizations of the standard model in F-theory. Though these models are Higgsable and the geometries are different, the results of those works suggest that similar chirality and hypercharge structures may also arise in our scenario.

\subsection{Abelian factors}

To obtain a realistic model containing (or exactly realizing) the standard model of particle physics, at least one U(1) factor must appear in the gauge group. Abelian gauge group factors are difficult to study in F-theory as they depend upon global features of the compactification; nonetheless, there has been significant recent progress in understanding these 
factors in the F-theory context [69, 77-90]. While it is possible to have non-Higgsable U(1) factors arising on manifolds that have a nonzero Mordell-Weil rank at all points in their moduli space, such as over certain bases with a $\mathbb{C}^{*}$-structure ("semi-toric" bases) that give a threefold related to the Schoen construction [49, 91, 92], these occur only on very special bases and have not yet been studied in 4 dimensional theories; we focus attention here on more generic Higgsable U(1) factors that can be tuned on most F-theory bases.

The main conclusion that we describe here is that while when $\mathrm{SU}(3) \times \mathrm{SU}(2)$ is realized in a Higgsable fashion the particles charged under an additional U(1) will not in general be localized at the intersection between the divisors carrying the nonabelian factors without special tuning (even for a $I V$ - $I I I$ intersection), when one of the nonabelian factors is non-Higgsable, in general there will always be $\mathrm{U}(1)$ charged matter localized at the intersection point.

It was shown in [69] that any Weierstrass model that admits a $U(1)$ factor can be written in the form

$$
\begin{aligned}
y^{2}= & x^{3}+\left(e_{1} e_{3}-b^{2} e_{0}-\frac{1}{3} e_{2}^{2}\right) x \\
& +\left(-e_{0} e_{3}^{2}+\frac{1}{3} e_{1} e_{2} e_{3}-\frac{2}{27} e_{2}^{3}+\frac{2}{3} b^{2} e_{0} e_{2}-\frac{1}{4} b^{2} e_{1}^{2}\right),
\end{aligned}
$$

where, as described further in [90], $b$ is a section of a line bundle associated with an effective divisor $X([b]=X)$, and $\left[e_{i}\right]=(i-4) K+(i-2) X$. By making a choice of $X$, we can tune a model with a $\mathrm{U}(1)$ factor and various other desired singularities by appropriately choosing the sections $b, e_{i}$. By tuning $b \rightarrow 0$ in any such model with a $\mathrm{U}(1)$ factor, the $\mathrm{U}(1)$ is enhanced to a nonabelian gauge factor associated with the divisor $e_{3}$. While in some cases the resulting model reaches a transition point associated with a $(4,6)$ singularity on a codimension one locus and is best treated as a superconformal field theory, the associated nonabelian model can often give a simple picture of the matter spectrum of the abelian theory. In particular, the matter charged under the resulting $U(1)$ is localized on the vanishing locus of $e_{3}$, and the charge can often be understood via adjoint Higgsing of an associated non-abelian model.

As a simple example, consider again a $6 \mathrm{D}$ theory on the base $\mathbb{P}^{2}$, where the gauge group $\mathrm{SU}(3) \times \mathrm{SU}(2)$ is realized through a (tuned, Higgsable) $I V-I I I$ intersection, with the gauge group factors on the loci $U=\{u=0\}, Z=\{z=0\}$, as described in section 5.1.3. The simplest choice of effective divisor for $X$ is the trivial class, so that $b$ is simply a constant. In this case, $\left[e_{3}\right]=-K$, so $e_{3}$ is a cubic. Tuning an $\mathrm{SU}(2)$ on a cubic, from anomaly cancellation there are 54 fundamentals under the SU(2) and one adjoint. Since generically a cubic crosses a line at three points, there are generically three matter multiplets in each of the $(3,1,2)$ and $(1,2,2)$ representations arising from the three intersection points of each type. Higgsing the adjoint breaks the $\mathrm{SU}(2)$, giving a $\mathrm{U}(1)$ theory with 54 pairs of $+1,-1$ charged matter fields, 15 of which carry charges under the remaining nonabelian factors, but none of which need to reside at the $I V-I I I$ intersection point, unless a special cubic is chosen that passes through that point. Indeed, 108 is the minimal number of charged matter fields that are compatible with anomaly cancellation for a theory with a single 
$\mathrm{U}(1)$ on $\mathbb{P}^{2}$ [78]. This illustrates the fact that in general when the $\mathrm{SU}(3)$ and $\mathrm{SU}(2)$ are Higgsable, matter charged under an additional U(1) need not reside at the intersection of the divisors carrying the nonabelian factors, so in particular matter charged under both the $\mathrm{SU}(3)$ and $\mathrm{SU}(2)$ factors will in general not carry a $\mathrm{U}(1)$ charge. A similar story holds for $4 \mathrm{D}$ models with a Higgsable $\mathrm{SU}(3) \times \mathrm{SU}(2)$.

When the $\mathrm{SU}(3)$ is non-Higgsable and there is a $\mathrm{U}(1)$, however, there will generally be $\mathrm{U}(1)$ charged matter at the intersection of the $\mathrm{SU}(3)$ and any nonabelian $\mathrm{SU}(2)$ factor. This follows from an analysis similar to that carried out in [90], which holds for F-theory models in four dimensions as well as in six. Basically, the idea is that if there is a non-Higgsable $\mathrm{SU}(3)$ supported on a divisor $D$ then $D$ appears as an irreducible component of $-K$ and can therefore appear as an irreducible component of $e_{3}=-K+X$. Since matter charged under the $\mathrm{U}(1)$ lies at the intersections of $e_{3}$ with other divisors carrying nonabelian gauge groups, there will then necessarily be matter charged under the $\mathrm{U}(1)$ at the $\mathrm{SU}(3) \times \mathrm{SU}(2)$ intersection. As an example, analogous to the $6 \mathrm{D}$ example described above, a simple way to arrange for a $\mathrm{U}(1)$ factor in the presence of a non-Higgsable type $I V$ singularity carrying an $\mathrm{SU}(3)$ along $\{u=0\}$ that intersects with an $\mathrm{SU}(2)$ on $\{z=0\}$ is to set $X=[b]=0$, so $b$ is simply a constant. Since $e_{3}, e_{2}, e_{1}$ are then in the divisor classes of $-K,-2 K,-3 K$, each must vanish to order at least one in $u$. We see then that in the $b \rightarrow 0$ limit, $g$ vanishes to order 3 , so we have an $I_{0}^{*}$ Kodaira type on $u=0$, generally associated with a rank enhancement. In principle, the charged matter content under the U(1) can be computed in any such model. The details will depend on the choice of $X$, but anytime there is a rank enhancement on the locus $u=0$ there will be additional charged matter at the intersection point that will acquire $\mathrm{U}(1)$ charge under the Higgsing corresponding to $b \neq 0$. We leave the details of such calculations for future work. Note, however, that if the $\mathrm{SU}(2)$ is also nonHiggsable, then in the case $X=0, e_{3}, e_{2}, e_{1}$ must also vanish on $\{z=0\}$, which would give a $(4,6)$ singularity at the intersection point $u=z=0$ in the limit $b \rightarrow 0$, corresponding to similar situations in [90] where the $\mathrm{U}(1)$ cannot be unhiggsed to a nonabelian factor without going to a superconformal point. Such a situation may be avoided by choosing $X$ to be effective and sufficiently large to reduce the vanishing of $e_{3}$ on $u, z$.

A particularly simple example of the preceding construction for a model with a $U(1)$ factor can be realized by taking $X$ to be the trivial class, so $b$ is a constant, and furthermore setting $e_{2}=e_{3}=0$. This can be done on any base, since $X=0$ is always an effective divisor. In this case, $e_{0}, e_{1}$ must contain $Z, U$ as components with minimal multiplicities giving the Weierstrass model

$$
y^{2}=x^{3}-b^{2} z u^{2} \tilde{e_{0}} x-\frac{1}{4} b^{2} z^{2} u^{2}{\tilde{e_{1}}}^{2} .
$$

This model has the type $I V-I I I$ intersection and a $\mathrm{U}(1)$ factor.

Finally, we comment briefly on charges. To get the precise U(1) hypercharges of the standard model, it is necessary to have different $\mathrm{U}(1)$ charges associated with matter in different representations of the nonabelian part of the gauge group. Such different charges can arise when $e_{3}$ contains one of the loci supporting the nonabelian gauge factors as a component. Higher charges can also arise when $e_{3}$ is a singular divisor. As discussed 
in $[75,90]$, higher symmetric representations of $\mathrm{SU}(2)$ can likely be tuned on sufficiently singular divisors in the base. When $e_{3}$ is such a divisor, we can locally view the $\mathrm{U}(1)$ as a broken nonabelian group, and higher symmetric representations of $\mathrm{SU}(2)$ will give rise to fields with larger charges under $\mathrm{U}(1)$. We leave a full analysis of different realizations of $\mathrm{U}(1)$ and matter charges in models with non-Higgsable QCD to further work, though we present a four-dimensional anomaly analysis in section 7 , which shows that the minimal (in a certain sense) chiral $\mathrm{SU}(3) \times \mathrm{SU}(2) \times \mathrm{U}(1)$ model contains families of standard model fermions.

\subsection{The the low-energy physical theory and G-flux}

As mentioned earlier, unlike in six-dimensional theories, for four-dimensional F-theory models the gauge group and matter content do not necessarily match precisely with those determined from the geometry. While in some cases the low-energy physics is not significantly modified from that described by the geometry, in other cases G-flux (or possible other mechanisms such as D3-branes) can modify the spectrum of the theory. We do not attempt a complete analysis of these issues here but make a few comments that may help in framing future work in this direction. A good review of some of the general issues associated with G-flux is given in [5].

From the point of view of F-theory as a limit of M-theory, G-flux corresponds to the four-form flux of the M-theory 3-form potential wrapped on nontrivial cycles in the (resolved) elliptically fibered Calabi-Yau fourfold $X$ on which M-theory is compactified. From the type IIB point of view, such fluxes can correspond either to three-form flux on the compactification space or fluxes in the world-volume of seven-branes. G-flux must satisfy several conditions. First, tadpole cancellation dictates that

$$
\chi(X) / 24=\int G \wedge G+N_{\mathrm{D} 3},
$$

where $\chi(X)$ is the Euler character of the fourfold $X$, and $N_{\mathrm{D} 3}$ is the number of D3-branes in the system. G-flux must also satisfy the parity condition [93]

$$
G+\frac{c_{2}}{2} \in H^{4}(X, \mathbb{Z})
$$

These conditions generally require nonzero G-flux, though if $c_{2} / 2 \in H^{4}(X, \mathbb{Z})$ it is possible to take $G=0$, satisfying (6.3) entirely by the inclusion of D3-branes. In general, for a given geometry the number of possible G-flux configurations that satisfy the conditions (6.3) and (6.4) is extremely large, which is famously helpful for the cosmological constant problem but problematic for any attempts to explicitly compute solutions on a case-by-case basis.

When G-flux is present, several effects can in principle change the spectrum of the theory, modifying the gauge group and matter content from that of a particular member in the family of Weierstrass models. The first issue is the superpotential $W=\int G \wedge \Omega$ [94]. In general, this superpotential stabilizes many of the moduli. If the moduli are stabilized at a generic point in the complex structure moduli space, then the gauge group is that described by the non-Higgsable clusters in the base. It is possible, however, that in some situations the superpotential may stabilize the moduli on a locus in moduli space with an enhanced gauge 
symmetry, so that the resulting $4 \mathrm{D}$ theory has a larger gauge symmetry than is indicated by the structure of non-Higgsable clusters. It seems plausible that for many choices of G-flux (and perhaps most or all) no such symmetry enhancement would occur, but it is a logical possibility that such a symmetry enhancement can be forced in certain situations.

A second way in which the gauge group can be modified is by turning on fluxes on the seven-brane world volume in the F-theory picture. Such fluxes can break the symmetry group supported on the seven-branes in question. While some specific examples have been identified where this occurs (see e.g. [22] and references therein), it is not clear how generally (given all possible flux choices) this can or must occur in models. From a practical point of view, however, constraints on the flux quanta that are required by the absence of flux breaking are to some extent understood; see e.g. [95]. In many known cases, particularly those with heterotic duals, solutions exist, and therefore there exist consistent choices of G-flux which do not break the geometrically-determined gauge group.

Finally, G-flux can affect the matter spectrum of the theory. In the absence of G-flux, the matter spectrum is purely non-chiral, " $\mathcal{N}=2$ " like matter. When G-flux is included, the matter spectrum generally develops a chiral component. In particular, the chiral index of states in a four-dimensional $\mathcal{N}=1 \mathrm{~F}$-theory compactification can be determined by the choice of $G$-flux in the related three-dimensional $\mathcal{N}=2$ M-theory compactification. Over the last few years this relationship has been explored in great detail; see for example [6163] for analyses of G-flux in global models in the context of spectral covers, [95, 96] for analyses based on the resolved geometry and M-theory, and [80] for an exploration of the relationship between this flux and anomaly cancellation in the four-dimensional F-theory compactification.

Understanding how these various aspects of G-flux interact with non-Higgsable structures in an F-theory compactification presents a variety of questions for further work. In the most straightforward scenario, to realize a realistic standard model using a non-Higgsable SU(3), we would want to identify an elliptically fibered CY fourfold with a non-Higgsable $\mathrm{SU}(3)$, and either a non-Higgsable or Higgsable $\mathrm{SU}(2)$, in a setup where G-flux neither enhanced or diminished the geometric F-theory gauge group (as often assumed in the Ftheory model building literature), but where the G-flux would give rise to the proper chiral spectrum for the standard model. The explicit examples given in section 5 could serve as a starting point for such an analysis.

As described in the next section, four-dimensional anomaly cancellation places fairly strong constraints on the resulting spectrum, so that multiple generations of standard model matter is one of the few simple solutions that is consistent with all known conditions. There are other possible scenarios, however, as well, in which non-Higgsable structures could play a role in a realistic F-theory construction of the standard model. For example, there could be a non-Higgsable $E_{6}$ GUT group that is broken by flux on the seven-branes that carry the group. Or, moduli stabilization from the F-theory superpotential could push the theory to a locus with an unbroken gauge group larger than the geometric non-Higgsable gauge group. We leave further investigation of these interesting questions to future work.

It is clear any case, however, that an important issue that must be understood more clearly to progress with the ideas that we have developed in this paper is the relationship 
between configurations in 4D F-theory geometry that are non-Higgsable from the point of view of complex structure moduli space and the physics of the corresponding low-energy 4D supergravity theories. In six-dimensional theories, gauge groups and matter that are geometrically non-Higgsable correspond to gauge groups and matter in the low-energy theory that are physically non-Higgsable. In these cases, the non-Higgsability of the theory can be understood from the point of view of low-energy field theory. For example, the matter in a $6 \mathrm{D}$ theory with a non-Higgsable $G_{2} \times \mathrm{SU}(2)$ cluster lives in a single half-hypermultiplet of the $\mathrm{SU}(2)$ (times a $\mathbf{7}+\mathbf{1}$ of the $G_{2}$ ), and cannot be Higgsed because, from the $\mathcal{N}=1$ point of view, the D-term constraints cannot cancel for a single fundamental of $\mathrm{SU}(2)$.

On the other hand, in many 4D models with geometrically non-Higgsable structure such as we have considered here, the associated matter fields appear to allow for D-flat symmetry breaking directions that may or may not be F-flat. In the framework of $d=4$ $\mathcal{N}=1$ Lagrangian supergravity, the absence of a superpotential which obstructs such a flat direction would correspond to a Higgsable theory; geometric non-Higgsability may provide evidence that such a superpotential must exist. As a concrete example, in a theory with fields $Q, D, L$ transforming in the representations $(\mathbf{3}, \mathbf{2}),(\overline{\mathbf{3}}, \mathbf{1}),(\mathbf{1}, \mathbf{2})$ of a group $\mathrm{SU}(3) \times \mathrm{SU}(2)$, the scalar combination $Q D L$ is gauge invariant and can in principle be turned on to Higgs the gauge group. In a theory where the group is geometrically nonHiggsable, these fields do not correspond to complex structure moduli of the corresponding elliptic fibration. The simplest interpretation of the geometric non-Higgsability would be that while the fields $Q, D, L$ may be massless at quadratic order, a cubic term $Q D L$ in the superpotential may stabilize these fields at quartic order. Indeed, in general without some explicit symmetry (such as R-parity) to forbid such terms, they are expected to arise from perturbative and/or nonperturbative effects. Understanding how this story works out in $4 \mathrm{D}$ F-theory constructions is an important question that we hope will be addressed by further work on these models, or more broadly on non-Higgsable clusters.

While the simplest and most satisfactory scenario might be that in those cases where the geometrically non-Higgsable gauge group and matter content persist in the low-energy $4 \mathrm{D}$ theory the non-Higgsability of the matter fields automatically arises from D-term and/or F-term constraints in the supergravity theory, we cannot rule out completely other possibilities. For example, there are additional degrees of freedom other than the complex structure moduli that are relevant in the low-energy $4 \mathrm{D}$ theory, but not yet fully understood in the context of F-theory. It may be possible that these degrees of freedom can give expectation values to matter fields which Higgs the gauge theory, even if complex structure deformations may not. Such degrees of freedom include continuous moduli associated with world volume gauge fields on the seven-branes in the IIB picture, or associated with $h^{2,1}$ of the Calabi-Yau fourfold in the M-theory picture.

At this point in time, F-theory is not a completely defined framework, and there is not yet a systematic way of constructing the complete low-energy supergravity theory associated with a given F-theory compactification. The configurations that contain nonHiggsable clusters necessarily involve nonperturbative physics from the string theory point of view, so new insights may be needed to clarify the physical mechanisms involved in vacuum solutions constructed using these geometries. Nevertheless, they are an interesting direction for current and future research. 


\section{Summary and discussion}

In this final section, we summarize the main results of the paper, make a brief but potentially significant argument from four-dimensional anomaly cancellation, and discuss some possible physical lessons.

\subsection{A summary of non-Higgsable QCD}

In this section we review some of the main results from sections 4 and 5 on the three qualitatively different realizations of non-Higgsable QCD and their associated non-abelian matter spectra; see table 4 . In section 3 we gave an overview of the possible ways in which the standard model could be realized in an F-theory compactification, emphasizing the possible role of non-Higgsable clusters. For much of the rest of the paper we have focused on "non-Higgsable QCD" in which the $\mathrm{SU}(3)$ factor in the standard model gauge group is a geometrically non-Higgsable gauge factor realized on a Kodaira type $I V$ codimension one singularity in the F-theory base.

We have shown that there are three qualitatively different possible realizations of nonHiggsable QCD, depending upon how the SU(2) factor of the standard model gauge group is realized. The three possibilities for the $\mathrm{SU}(2)$ are from seven-branes associated to a type $I V$ fiber with outer monodromy, a type $I I I$ fiber, or an $I_{2}$ fiber. We refer to these scenarios as $I V-I V m, I V-I I I$, and $I V-I_{2}$, respectively. In each case we have analyzed the geometric matter content along the curve in $B_{3}$ lying at the intersection of the divisors supporting the $\mathrm{SU}(3)$ and $\mathrm{SU}(2)$ gauge group factors. This determines the Lie algebra representations, but gives matter that is non-chiral in the absence of G-flux; as such, G-flux must be incorporated to produce chiral matter as described in section 6.2. The multiplicity (number of generations) of matter will also be a model-dependent quantity that must be analyzed separately in specific examples. Here we briefly summarize the Lie algebraic (geometric) structure of matter in each of the three scenarios. In each case we have identified in section 5 some specific examples of (toric) threefold bases $B_{3}$ in which the different types of non-Higgsable and Higgsable $\mathrm{SU}(3)$ and $\mathrm{SU}(2)$ group factors are realized. These examples serve as existence proofs for the general ideas described in the paper, and as useful starting points for further analysis.

The IV-III case. The possibility of $I V-I I I$ non-Higgsable QCD was introduced in section 4, including a string junction analysis of two-cycles in an elliptic surface near the $I V-I I I$ intersection. One interesting feature is that an extra brane which carries an $I_{1}$ singularity is always present at this intersection, and string junctions may end on this brane. Together with the anomaly analysis of section 5 , we found that the spectrum of matter representations on the $I V-I I I$ intersection curve that transform in nontrivial $\mathrm{SU}(3) \times \mathrm{SU}(2)$ representations is (denoting the fundamental of $\mathrm{SU}(N)$ as $\square_{N}$ ),

$$
\left(\square 3, \square_{2}\right)+\square_{3}+\square_{2}
$$

which is precisely the set of non-trivial $\mathrm{SU}(3) \times \mathrm{SU}(2)$ representations that are realized by matter fields in the standard model (up to conjugates, which are automatically included at the geometric level). The string junction analysis also showed the existence of $\mathrm{SU}(3) \times \mathrm{SU}(2)$ 


\begin{tabular}{|cc|}
\hline Realization & Non-trivial $\mathrm{SU}(3) \times \mathrm{SU}(2)$ Spectrum \\
\hline$I V-I I I$ & $\left(\square_{3}, \square_{2}\right)+\square_{3}+\square_{2}$ \\
$I V-I V m$ & $\left(\square_{3}, \square_{2}\right)+\square_{3}$ \\
$I V-I_{2}$ & $\left(\square, \square_{3}\right)+\square$ \\
\hline
\end{tabular}

Table 4. The non-trivial SU(3) $\times \mathrm{SU}(2)$ representations at the collision of SU(3) and SU(2) sevenbranes for each of the three realizations of non-Higgsable QCD.

singlet states on the same matter curve; such singlets embed into the intermediate 16 of $\mathrm{SO}(10)$ (realized on the extra brane) as expected of right-handed electrons or neutrinos. As discussed in section 6, when an extra $\mathrm{U}(1)$ factor is incorporated in a model of this type, matter at the intersection point acquires charges under the $\mathrm{U}(1)$, and the presence of G-flux produces a chiral matter spectrum. As we will show shortly, anomaly cancellation then strongly restricts the set of possible multiplicities and $\mathrm{U}(1)$ charges, so that in a simple picture where there are multiple generations of a common matter structure, the set of fields in the standard model is one of the only possible consistent solutions.

The $\boldsymbol{I} \boldsymbol{V}-\boldsymbol{I} \boldsymbol{V} \boldsymbol{m}$ and $\boldsymbol{I} \boldsymbol{V}-\boldsymbol{I}_{\mathbf{2}}$ cases. In the remaining cases, the $\mathrm{SU}(2)$ can be Higgsable or non-Higgsable when realized on a type $I V$ singularity with monodromy, and must be Higgsable when realized on a type $I_{2}$ singularity. In section 5 we performed an anomaly analysis similar to the one for the $I V-I I I$ case in order to determine the $\mathrm{SU}(3) \times \mathrm{SU}(2)$ matter representations at the associated seven-brane collisions. In both cases we have found that the non-trivial $\mathrm{SU}(3) \times \mathrm{SU}(2)$ spectrum at the collision of the $\mathrm{SU}(3)$ and $\mathrm{SU}(2)$ seven-branes is

$$
\left(\square_{3}, \square_{2}\right)+\square_{3} .
$$

Notably, the $\square_{2}$ that is present at the $I V-I I I$ collision is not present at the $I V-I V m$ or $I V-I_{2}$ collision. This makes these scenarios potentially less attractive for realizing the full standard model spectrum without incorporating some additional structure.

\subsection{The minimal chiral $\mathrm{SU}(3) \times \mathrm{SU}(2) \times \mathrm{U}(1)$ model gives standard model generations}

Anomaly cancellation in four dimensions constrains the possible chiral models that one might obtain from non-Higgsable QCD. We briefly discuss this here, since it demonstrates the plausibility of obtaining chiral standard models in concrete four-dimensional nonHiggsable QCD models with G-flux. This analysis is similar to well-known results in the field theory literature, though presented here from the point of view of non-Higgsable QCD.

From the three geometric realizations of non-Higgsable QCD, we know that the allowed matter representations are $(\mathbf{3}, \mathbf{2}),(\mathbf{3}, \mathbf{1}),(\mathbf{1}, \mathbf{2})$, the conjugates of these representations, and the singlet representation. In a chiral four-dimensional model, $\mathrm{SU}(3)$ anomaly cancellation implies that the number of $\mathbf{3}$ and $\overline{\mathbf{3}}$ fields must match, so that if we have $N$ chiral $(\mathbf{3}, \mathbf{2})$ matter fields, we must have ${ }^{4}$ a corresponding $2 N(\overline{\mathbf{3}}, \mathbf{1})$ fields, suggesting $N$ generations

\footnotetext{
${ }^{4}$ In many cases it is known [80] that a consistent choice of G-flux automatically ensures anomaly cancellation.
} 
each with one $(\mathbf{3}, \mathbf{2})$ and two copies of $(\mathbf{3}, \mathbf{2})$. The number $K$ of $(\mathbf{1}, \mathbf{2})$ chiral fermions (multiplets) is not as precisely fixed since cubic $\mathrm{SU}(2)$ non-abelian anomaly cancellation is trivially satisfied. ${ }^{5}$ In the absence of additional chiral $\mathrm{SU}(2)$ fermions, however, the global $\mathrm{SU}(2)$ anomaly [98] requires $(3 N+K) \equiv 0(\bmod 2)$. We take as an assumption, though it is perhaps also natural to expect, that these $(\mathbf{1}, \mathbf{2})$ multiplets (if they exist) might arise in a family with the other fields. That is, we take $K=P N$ where $P$ is constrained by the global anomaly depending on $N$. This yields ${ }^{6} N$ copies of $(\mathbf{3}, \mathbf{2})+2 \times(\overline{\mathbf{3}}, \mathbf{1})+P \times(\mathbf{1}, \mathbf{2})+N_{S} \times(\mathbf{1}, \mathbf{1})$, where $N_{S}$ is the number of $\mathrm{SU}(3) \times \mathrm{SU}(2)$ singlets per family.

Given this structure, we would like to determine the minimal consistent chiral $\mathrm{SU}(3) \times$ $\mathrm{SU}(2) \times \mathrm{U}(1)$ model, as determined by the minimal $P$ and $N_{S}$. For $P=0$, the only anomaly free $\mathrm{U}(1)$ is the non-chiral $\mathrm{U}(1)$ that assigns opposite charges to the two copies of right-handed quarks; so to have a chiral model we must proceed to $P=1$. For $P=1$ and $N_{S}=0$, the only anomaly free $\mathrm{U}(1)$ is the same non-chiral U(1) just mentioned; this exists for any value of $N_{S}$. At $P=N_{S}=1$, however, there is another anomaly free $\mathrm{U}(1)$. It is chiral and has charges (denoted as subscripts)

$$
(\mathbf{3}, \mathbf{2})_{1}+(\overline{\mathbf{3}}, \mathbf{1})_{-4}+(\overline{\mathbf{3}}, \mathbf{1})_{2}+(\mathbf{1}, \mathbf{2})_{-3}+(\mathbf{1}, \mathbf{1})_{6} .
$$

This is precisely a generation of standard model fermions; the minimal chiral $\mathrm{U}(1)$ is the weak hypercharge. In summary, we see that the minimal chiral $\mathrm{SU}(3) \times \mathrm{SU}(2) \times \mathrm{U}(1)$ model that may arise from non-Higgsable QCD gives rise to families of standard model fermions.

\subsection{Discussion}

We have found that incorporating a non-Higgsable $\mathrm{SU}(3)$ or $\mathrm{SU}(3) \times \mathrm{SU}(2)$ into an F-theory compactification leads to a scenario in which certain features of the standard model, such as the unbroken QCD sector and the standard model matter spectrum, seem very natural. It is interesting and perhaps suggestive that $\mathrm{SU}(3), \mathrm{SU}(2)$, and $\mathrm{SU}(3) \times \mathrm{SU}(2)$ can be realized in a non-Higgsable fashion in F-theory, while $\mathrm{SU}(n)$ for $n>3$ cannot be realized in this way. We conclude this paper with some speculations regarding the physical implications of such a scenario, and also some comparison to related ideas in the literature.

Enhanced symmetry points in the landscape. Scenarios $[99,100]$ for moduli stabilization in type IIB string theory have led to a general consensus that there should exist a large landscape of metastable string vacua [101-104]. The existence of this large set of possible vacuum solutions prompts a number of interesting physical questions - for example, whether there exist identifiable vacua with the same properties as those we observe in nature, or whether there exists a dynamical mechanism for vacuum selection.

A related question is why, in a theory that can be characterized in terms of a large space of possible scalar field values with many metastable vacua distributed throughout it, one might expect stabilization to occur at a point with enhanced symmetry. More specifically, why would the scalar potential conspire to give rise to metastable vacua at enhanced symmetry points, and what dynamical mechanism might drive the moduli toward the enhanced

\footnotetext{
${ }^{5}$ Though in some contexts in the landscape, there are additional constraints on $\mathrm{SU}(2)$ theories related to brane nucleation [97].

${ }^{6}$ We emphasize that this is a statement about chiral matter; in some regions of moduli space there may be additional vector pairs, which could be interpreted as Higgs doublets.
} 
symmetry point? This question is interesting because of the symmetries or near-symmetries observed in our vacuum, and also the common expectation that a generic metastable vacuum in the landscape is not at an enhanced symmetry point. A number of works have studied this issue and demonstrated the existence of mechanisms that can trap moduli at enhanced symmetry points; see for example [10] and references therein, and also [105, 106].

One of the messages of this paper is that non-Higgsable clusters could provide an alternative answer to the same question. As opposed to explaining why moduli stabilization selects a vacuum at a special point in moduli space with enhanced symmetry, as in other approaches, non-Higgsable clusters circumvent the problem completely, since for geometries with one or more non-Higgsable clusters, a generic point in the moduli space has enhanced symmetry. Moduli stabilization must still occur, of course, but stabilization at a generic point is sufficient to obtain enhanced symmetry.

Based on what is currently known about non-Higgsable clusters and the structure of elliptically fibered Calabi-Yau manifolds, we believe that a common assumption about the landscape must be revisited. The assumption, used for example in the first approach described above, is that vacua with enhanced symmetry or light particles comprise a very small subset of the space of all metastable vacua. This assumption seems to be valid only to the extent that non-Higgsable clusters are non-generic in the landscape. F-theory, however, seems to provide at this point the largest range of possible string theory vacuum solutions, and seems to generically produce vacua containing non-Higgsable clusters.

From what is currently understood, in fact, it appears that the overwhelming majority of F-theory vacua are likely constructed from Calabi-Yau manifolds that have one or more non-Higgsable clusters, which may mean that symmetries are in fact generic and a large part of the landscape. More specifically, for Calabi-Yau threefolds the complete set of toric bases $B_{2}$ and the larger set of "semi-toric" bases were analyzed in $[48,49,107]$. Out of all these bases, the vast majority had multiple non-Higgsable clusters, largely consisting of $E_{8}, F_{4}$, and $\mathrm{SU}(2) \times G_{2}$ clusters with minimal matter. For example, of more than 60,000 toric bases, only 16 (the toric del Pezzo and generalized del Pezzo surfaces) lacked non-Higgsable clusters. While the number of distinct Calabi-Yau manifolds that can be constructed as ("tuned") elliptic fibrations (see e.g. [37]) over the base is larger for the simpler bases that lack non-Higgsable clusters, such bases also have smaller Hodge numbers and have moduli spaces that are in some sense "smaller". In general, bases with larger Hodge numbers tend to have more non-Higgsable clusters, associated with large non-Higgsable gauge groups with many factors of the factors mentioned above.

For four-dimensional F-theory constructions, all indications are that a similarly vast majority of all allowed threefold bases give non-Higgsable clusters. Furthermore, those Calabi-Yau fourfolds with many non-Higgsable clusters tend to be those that have large Hodge numbers and therefore a larger number of expected stabilized flux vacua. It has been noted previously in the literature that compactifications on fourfolds with large Hodge numbers give large gauge groups [108]. Non-Higgsable clusters appear in other approaches to string compactification as well as F-theory. In [22], the set of toric threefold bases $B_{3}$ was constructed that give F-theory compactifications having dual heterotic descriptions on smooth elliptically fibered Calabi-Yau threefolds with smooth gauge bundles. Of these constructions the majority (roughly 85\%) exhibited non-Higgsable clusters. Furthermore, 
these constructions correspond precisely to those that have the least potential for nonHiggsable structure; the rest of the examples in a much larger class of bases with a similar structure as $\mathbb{P}^{1}$ bundles over a general toric base [50] generically exhibit non-Higgsable structures corresponding to singularities in the Calabi-Yau threefold carrying enhanced gauge symmetries on the heterotic side.

Thus, from our current understanding it seems quite plausible that the landscape is overwhelmingly dominated by vacua exhibiting symmetry. In such a scenario, the kind of non-Higgsable QCD model which we introduced in this paper may be a promising approach to realizing observed physics in a natural way a generic points in the string landscape. More broadly, non-Higgsable clusters may play an important role in determining the structure of symmetries and light particles at low energy scales. Given the apparent genericity of non-Higgsable clusters in the landscape, it is worth noting that they also may play other important roles, such as for supersymmetry breaking or dark matter phenomenology.

Non-Higgsable QCD and model building. The existence of an unbroken QCD sector is an experimental fact that also motivates the kind of scenario we have considered in this paper. This is a particularly important fact since if QCD were broken at a scale much higher than $\Lambda_{\mathrm{QCD}} \simeq 200 \mathrm{MeV}$, quark confinement would not occur and protons would cease to exist, changing physics drastically in a way not amenable to life as we know it. In the standard model the existence of an unbroken QCD sector is a simple artifact of the theory; it has no colored scalar. On the other hand, in four-dimensional theories with $\mathcal{N}=1$ supersymmetry the existence of an unbroken QCD sector must be accounted for, as such theories necessarily have colored scalars. This constrains model-building.

For example, in the minimal supersymmetric standard model (MSSM) there are supersymmetric color-breaking (nearly) flat directions; see [109] for a classification and [110] for an analytic study of the electroweak vacuum moduli space. The possible existence of color-breaking vacua constrains the soft breaking parameters. For example, the so-called A-terms are scalar trilinear soft breaking terms, and there are upper bounds on some of these parameters necessary for the absence of dangerous color breaking vacua; see for example $[111,112]$ and references therein. The term for the top quark $A_{t}$ appears in loop corrections to the mass of the Higgs boson, and $A_{t}$ must often be large in order to account for its observed value. Together, these two constraints can place significant tension on models; see e.g. [113] for one recent account in the CMSSM.

In the scenario we have proposed, the existence of an unbroken QCD sector is a natural possibility when model-building with non-Higgsable clusters in F-theory. As we have emphasized, $\mathrm{SU}(3)$ and $\mathrm{SU}(2)$ are the only $\mathrm{SU}(n)$ groups that may be realized through a non-Higgsable cluster. We have considered the possibility that $\mathrm{SU}(3)_{Q C D}$ is realized as a non-Higgsable cluster, which requires a non-perturbative seven-brane realized by a type $I V$ fiber. We looked in particular detail at one geometry, obtained from the intersection of a type $I V$ and a type $I I I$ fiber.

There are a number of interesting model-building implications of non-Higgsable clusters. First, conventional SU(5) grand unification cannot be realized at a generic point in the moduli space of a compactification with a non-Higgsable cluster; specialization in moduli space is necessary. $E_{6}$ or $\mathrm{SO}(10)$ grand unification may be possible with a non-Higgsable cluster, however, as discussed in section 3, with $\mathrm{SO}(10)$ also requiring specialization. 
Second, though we have classified in broad strokes the possible realizations of a nonHiggsable QCD sector in F-theory, we have not performed a detailed analysis of all of the geometries. Such an analysis may imply the existence of potentially interesting exotics that are experimentally allowed and potentially discoverable at the LHC. Exotics are known to be a feature in many corners of the landscape and their existence can sometimes be related to string consistency conditions that do not have a simple analog in quantum field theory; see e.g. $[97,114]$ for a study of how stringy constraints can imply the existence of quasichiral electroweak exotics. More generally, while anomalies can act as a low-energy window on constraints that must be satisfied by any UV completion of a quantum theory, the geometry of F-theory vacua may place further specific constraints on the set of low-energy fields and interactions available in the $4 \mathrm{D}$ theory in ways that are not yet understood from the lowenergy point of view. Such considerations have led to a productive line of inquiry in the context of six-dimensional theories (see e.g. [74, 115]); an initial investigation of possible constraints that may arise in this fashion for four-dimensional theories was carried out in [47].

Finally, while our scenario requires the existence of a non-Higgsable QCD sector, we leave open the possibility that there exist other non-Higgsable gauge factors. As mentioned above, an interesting possibility is that of a non-Higgsable hidden sector, which may give rise to interesting dark matter or supersymmetry breaking phenomenology. Also, given that $\mathrm{SU}(2)$ and $\mathrm{U}(1)$ are gauge factors that may also be non-Higgsable, it would be interesting to study the possibility of a non-Higgsable electroweak sector. At first glance this seems to be ruled out experimentally, but given the successes of radiative electroweak symmetry breaking in other theories it may be worth considering. If possible it would likely, as in the MSSM, depend heavily on the details of supersymmetry breaking and renormalization group flow. In one sense it would also appeal to minimality; only ten individual group factors may be realized by non-Higgsable seven-branes, and it so happens that the three factors of lowest dimension are precisely $\mathrm{SU}(3), \mathrm{SU}(2)$, and $\mathrm{U}(1)$.

\section{Acknowledgments}

We thank Lara Anderson, Mirjam Cvetič, Keshav Dasgupta, Tony Gherghetta, James Gray, Tom Hartman, Kristan Jensen, Sam Johnson, Denis Klevers, Paul Langacker, Liam McAllister, Paul McGuirk, Dave Morrison, Daniel Park, Joe Polchinski, and Jesse Thaler for useful conversations. J.H. thanks J.L. Halverson for her kind support and constant encouragement. The research of J.H. was supported in part by the National Science Foundation under Grant No. PHY11-25915. The research of W.T. is supported by the U.S. Department of Energy under grant Contract Number DE-SC00012567. J.L.S. is supported by DARPA, fund no. 553700, and is the Class of 1939 Professor in the Schools of Arts and Sciences of the University of Pennsylvania; he gratefully acknowledges the generosity of the Class of 1939. A.G. is supported by NSF Research Traning Group Grant GMS0636606. We thank the Aspen Center for Physics for hospitality and partial support by the National Science Foundation Grant No. PHYS-1066293. We also thank the Simons Center for Geometry and Physics for hospitality while this work was carried out. 


\begin{tabular}{|ccc|}
\hline Junction & Dynkin Labels & Representation \\
\hline$(-1,-1,1,1,0,-1,1,0)$ & $(0,0,0)$ & Singlet \\
\hline$(-1,0,1,0,-1,-1,0,-1)$ & $(0,0,0)$ & Singlet \\
\hline$(-1,0,1,1,0,0,0,-1)$ & $(1,0,1)$ & $(\mathbf{3}, \mathbf{2})$ \\
$(-1,0,1,1,0,-1,0,0)$ & $(-1,1,1)$ & \\
$(-1,-1,0,0,0,0,0,-1)$ & $(1,0,-1)$ & \\
$(-1,0,1,1,-1,-1,1,0)$ & $(0,-1,1)$ & \\
$(-1,-1,0,0,0,-1,0,0)$ & $(-1,1,-1)$ & \\
$(-1,-1,0,0,-1,-1,1,0)$ & $(0,-1,-1)$ & $(\overline{\mathbf{3}}, \mathbf{1})$ \\
\hline$(-1,-1,0,1,1,0,0,0)$ & $(0,1,0)$ & \\
$(-1,-1,0,1,0,0,1,0)$ & $(1,-1,0)$ & $(\overline{\mathbf{3}}, \mathbf{1})$ \\
$(-1,-1,0,1,0,-1,1,1)$ & $(-1,0,0)$ & \\
\hline$(-1,0,0,0,0,0,-1,-1)$ & $(0,1,0)$ & $(\mathbf{1}, \mathbf{2})$ \\
$(-1,0,0,0,-1,0,0,-1)$ & $(1,-1,0)$ & \\
$(-1,0,0,0,-1,-1,0,0)$ & $(-1,0,0)$ & \\
\hline$(-1,0,0,1,0,0,0,0)$ & $(0,0,1)$ & \\
$(-1,-1,-1,0,0,0,0,0)$ & $(0,0,-1)$ & \\
\hline$(0,0,1,1,1,0,0,0)$ & $(0,1,1)$ & \\
$(0,0,1,1,0,0,1,0)$ & $(1,-1,1)$ & \\
$(0,-1,0,0,1,0,0,0)$ & $(0,1,-1)$ & \\
$(0,0,1,1,0,-1,1,1)$ & $(-1,0,1)$ & \\
$(0,-1,0,0,0,0,1,0)$ & $(1,-1,-1)$ & \\
$(0,-1,0,0,0,-1,1,1)$ & $(-1,0,-1)$ & \\
\hline$(0,1,1,0,0,0,-1,-1)$ & $(0,1,1)$ & \\
$(0,1,1,0,-1,0,0,-1)$ & $(1,-1,1)$ & \\
$(0,0,0,-1,0,0,-1,-1)$ & $(0,1,-1)$ & \\
$(0,1,1,0,-1,-1,0,0)$ & $(-1,0,1)$ & \\
$(0,0,0,-1,-1,0,0,-1)$ & $(1,-1,-1)$ & \\
$(0,0,0,-1,-1,-1,0,0)$ & $(-1,0,-1)$ & \\
\hline$(0,0,1,0,0,0,0,-1)$ & $(1,0,0)$ & \\
$(0,0,1,0,0,-1,0,0)$ & $(-1,1,0)$ & \\
$(0,0,1,0,-1,-1,1,0)$ & $(0,-1,0)$ & \\
\hline$(0,-1,0,1,1,0,1,1)$ & $(0,0,0)$ & \\
\hline$(0,0,0,0,1,1,-1,-1)$ & $(1,1,0)$ & \\
$(0,0,0,0,1,0,-1,0)$ & $(-1,2,0)$ & \\
$(0,0,0,0,0,1,0,-1)$ & $(2,-1,0)$ & \\
\hline$(0,1,1,1,0,0,0,0)$ & $(0,0,2)$ & \\
\hline
\end{tabular}

Table 5. Displayed are half the string junctions in an elliptic surface near the $I V$ - $I I I$ collision; group theoretically, these are the 36 positive roots in an adjoint of $E_{6}$. We also display their representation under $\mathrm{SU}(3) \times \mathrm{SU}(2)$ and corresponding Dynkin labels; in the latter, the first two entries and last entry are the $\mathrm{SU}(3)$ and $\mathrm{SU}(2)$ Dynkin labels, respectively. The first sixteen junctions end on the extra brane and are in the same $\mathrm{SU}(3) \times \mathrm{SU}(2)$ representations as a generation of standard model fermions. The relationship of this data to the matter spectrum at the $I V$-III collision is described in detail in the text; they are related but not identical, since we have only performed a partial deformation analysis. 
Open Access. This article is distributed under the terms of the Creative Commons Attribution License (CC-BY 4.0), which permits any use, distribution and reproduction in any medium, provided the original author(s) and source are credited.

\section{References}

[1] C. Vafa, Evidence for F-theory, Nucl. Phys. B 469 (1996) 403 [hep-th/9602022] [inSPIRE].

[2] D.R. Morrison and C. Vafa, Compactifications of F-theory on Calabi-Yau threefolds. 1, Nucl. Phys. B 473 (1996) 74 [hep-th/9602114] [INSPIRE].

[3] D.R. Morrison and C. Vafa, Compactifications of F-theory on Calabi-Yau threefolds. 2, Nucl. Phys. B 476 (1996) 437 [hep-th/9603161] [INSPIRE].

[4] D.R. Morrison, TASI lectures on compactification and duality, hep-th/0411120 [INSPIRE].

[5] F. Denef, Les Houches lectures on constructing string vacua, arXiv:0803.1194 [INSPIRE].

[6] W. Taylor, TASI lectures on supergravity and string vacua in various dimensions, arXiv:1104.2051 [INSPIRE].

[7] J.J. Heckman, Particle physics implications of F-theory, Ann. Rev. Nucl. Part. Sci. 60 (2010) 237 [arXiv:1001.0577] [INSPIRE].

[8] T. Weigand, Lectures on F-theory compactifications and model building, Class. Quant. Grav. 27 (2010) 214004 [arXiv:1009.3497] [INSPIRE].

[9] D.R. Morrison and W. Taylor, Classifying bases for 6D F-theory models, Central Eur. J. Phys. 10 (2012) 1072 [arXiv:1201.1943] [INSPIRE].

[10] L. Kofman et al., Beauty is attractive: moduli trapping at enhanced symmetry points, JHEP 05 (2004) 030 [hep-th/0403001] [INSPIRE].

[11] N. Nakayama, On Weierstrass models, in Algebraic geometry and commutative algebra, vol. II, Kinokuniya, Tokyo Japan (1988), pg. 405.

[12] K. Kodaira, On compact analytic surfaces. II, Ann. Math. 77 (1963) 563.

[13] K. Kodaira, On compact analytic surfaces. III, Ann. Math. 78 (1963) 1.

[14] N. Seiberg and E. Witten, Comments on string dynamics in six-dimensions, Nucl. Phys. B 471 (1996) 121 [hep-th/9603003] [InSPIRE].

[15] N. Seiberg, Nontrivial fixed points of the renormalization group in six-dimensions, Phys. Lett. B 390 (1997) 169 [hep-th/9609161] [INSPIRE].

[16] J.J. Heckman, D.R. Morrison and C. Vafa, On the classification of $6 D$ SCFTs and generalized ADE orbifolds, JHEP 05 (2014) 028 [arXiv:1312.5746] [INSPIRE].

[17] M. Del Zotto, J.J. Heckman, A. Tomasiello and C. Vafa, 6D conformal matter, arXiv: 1407.6359 [INSPIRE].

[18] K. Ohmori, H. Shimizu, Y. Tachikawa and K. Yonekura, Anomaly polynomial of general $6 D$ SCFTs, Prog. Theor. Exp. Phys. 2014 (2014) 103B07 [arXiv:1408.5572] [InSPIRE].

[19] K. Intriligator, $6 D, N=(1,0)$ Coulomb branch anomaly matching, JHEP 10 (2014) 162 [arXiv: 1408.6745] [INSPIRE].

[20] M. Bershadsky et al., Geometric singularities and enhanced gauge symmetries, Nucl. Phys. B 481 (1996) 215 [hep-th/9605200] [INSPIRE]. 
[21] S. Katz, D.R. Morrison, S. Schäfer-Nameki and J. Sully, Tate's algorithm and F-theory, JHEP 08 (2011) 094 [arXiv:1106.3854] [InSPIRE].

[22] L.B. Anderson and W. Taylor, Geometric constraints in dual F-theory and heterotic string compactifications, JHEP 08 (2014) 025 [arXiv: 1405.2074] [INSPIRE].

[23] D.R. Morrison and W. Taylor, Non-Higgsable clusters for $4 D$ F-theory models, arXiv: 1412.6112 [INSPIRE].

[24] J.H. Schwarz, An SL(2,Z) multiplet of type IIB superstrings, Phys. Lett. B 360 (1995) 13 [Erratum ibid. B 364 (1995) 252] [hep-th/9508143] [INSPIRE].

[25] M.R. Gaberdiel and B. Zwiebach, Exceptional groups from open strings, Nucl. Phys. B 518 (1998) 151 [hep-th/9709013] [INSPIRE].

[26] O. DeWolfe and B. Zwiebach, String junctions for arbitrary Lie algebra representations, Nucl. Phys. B 541 (1999) 509 [hep-th/9804210] [inSPIRE].

[27] A. Grassi, J. Halverson and J.L. Shaneson, Matter from geometry without resolution, JHEP 10 (2013) 205 [arXiv:1306.1832] [INSPIRE].

[28] A. Grassi, J. Halverson and J.L. Shaneson, Non-Abelian gauge symmetry and the Higgs mechanism in F-theory, arXiv: 1402.5962 [INSPIRE].

[29] S.H. Katz and C. Vafa, Matter from geometry, Nucl. Phys. B 497 (1997) 146 [hep-th/9606086] [INSPIRE].

[30] A. Grassi and D.R. Morrison, Group representations and the Euler characteristic of elliptically fibered Calabi-Yau threefolds, math/0005196 [INSPIRE].

[31] D.R. Morrison and W. Taylor, Matter and singularities, JHEP 01 (2012) 022 [arXiv:1106.3563] [INSPIRE].

[32] M. Esole and S.-T. Yau, Small resolutions of SU(5)-models in F-theory, Adv. Theor. Math. Phys. 17 (2013) 1195 [arXiv:1107.0733] [INSPIRE].

[33] A. Grassi and D.R. Morrison, Anomalies and the Euler characteristic of elliptic Calabi-Yau threefolds, Commun. Num. Theor. Phys. 6 (2012) 51 [arXiv:1109.0042] [inSPIRE].

[34] C. Lawrie and S. Schäfer-Nameki, The Tate form on steroids: resolution and higher codimension fibers, JHEP 04 (2013) 061 [arXiv:1212.2949] [INSPIRE].

[35] H. Hayashi, C. Lawrie, D.R. Morrison and S. Schäfer-Nameki, Box graphs and singular fibers, JHEP 05 (2014) 048 [arXiv: 1402.2653] [INSPIRE].

[36] M. Esole, S.-H. Shao and S.-T. Yau, Singularities and gauge theory phases, arXiv: 1402.6331 [INSPIRE].

[37] S.B. Johnson and W. Taylor, Calabi-Yau threefolds with large $h^{2,1}$, JHEP 1410 (2014) 23 [arXiv: 1406.0514] [INSPIRE].

[38] V. Batyrev, Variations of the mixed Hodge structure of affine hypersurfaces in algebraic tori, Duke Math. J. 69 (1993) 349.

[39] V.V. Batyrev, Dual polyhedra and mirror symmetry for Calabi-Yau hypersurfaces in toric varieties, J. Alg. Geom. 3 (1994) 493 [alg-geom/9310003] [INSPIRE].

[40] M. Kreuzer and H. Skarke, Calabi-Yau four folds and toric fibrations, J. Geom. Phys. 26 (1998) 272 [hep-th/9701175] [INSPIRE]. 
[41] M. Lynker, R. Schimmrigk and A. Wisskirchen, Landau-Ginzburg vacua of string, M-theory and F-theory at $c=12$, Nucl. Phys. B 550 (1999) 123 [hep-th/9812195] [INSPIRE].

[42] P. Candelas, A. Constantin and H. Skarke, An abundance of K3 fibrations from polyhedra with interchangeable parts, Commun. Math. Phys. 324 (2013) 937 [arXiv:1207.4792] [INSPIRE].

[43] A. Klemm, B. Lian, S.S. Roan and S.-T. Yau, Calabi-Yau fourfolds for M-theory and F-theory compactifications, Nucl. Phys. B 518 (1998) 515 [hep-th/9701023] [INSPIRE].

[44] P. Berglund and P. Mayr, Heterotic string/F-theory duality from mirror symmetry, Adv. Theor. Math. Phys. 2 (1999) 1307 [hep-th/9811217] [INSPIRE].

[45] K. Mohri, F-theory vacua in four-dimensions and toric threefolds, Int. J. Mod. Phys. A 14 (1999) 845 [hep-th/9701147] [INSPIRE].

[46] A. Grassi, Divisors on elliptic Calabi-Yau four folds and the superpotential in F-theory. 1, J. Geom. Phys. 28 (1998) 289 [InSPIRE].

[47] T.W. Grimm and W. Taylor, Structure in $6 D$ and $4 D N=1$ supergravity theories from F-theory, JHEP 10 (2012) 105 [arXiv: 1204.3092] [INSPIRE].

[48] D.R. Morrison and W. Taylor, Toric bases for $6 D$ F-theory models, Fortsch. Phys. 60 (2012) 1187 [arXiv:1204.0283] [INSPIRE].

[49] G. Martini and W. Taylor, 6D F-theory models and elliptically fibered Calabi-Yau threefolds over semi-toric base surfaces, arXiv:1404.6300 [INSPIRE].

[50] J. Halverson and W. Taylor, to appear.

[51] J. Gray, A.S. Haupt and A. Lukas, All complete intersection Calabi-Yau four-folds, JHEP 07 (2013) 070 [arXiv:1303.1832] [INSPIRE].

[52] J. Gray, A.S. Haupt and A. Lukas, Topological invariants and fibration structure of complete intersection Calabi-Yau four-folds, JHEP 09 (2014) 093 [arXiv:1405.2073] [INSPIRE].

[53] K. Matsuki, Introduction to the Mori program, Springer-Verlag, Berlin Germany (2002).

[54] A. Grassi, On minimal models of elliptic threefolds, Math. Ann. 290 (1991) 287.

[55] J. Knapp and M. Kreuzer, Toric methods in F-theory model building, Adv. High Energy Phys. 2011 (2011) 513436 [arXiv:1103.3358] [INSPIRE].

[56] W. Fulton, Introduction to toric varieties, Annals of Mathematics Study 131, Princeton University Press, Princeton U.S.A. (1993).

[57] R. Donagi and M. Wijnholt, Model building with F-theory, Adv. Theor. Math. Phys. 15 (2011) 1237 [arXiv:0802.2969] [INSPIRE].

[58] C. Beasley, J.J. Heckman and C. Vafa, GUTs and exceptional branes in F-theory - I, JHEP 01 (2009) 058 [arXiv:0802.3391] [INSPIRE].

[59] C. Beasley, J.J. Heckman and C. Vafa, GUTs and exceptional branes in F-theory - II: experimental predictions, JHEP 01 (2009) 059 [arXiv:0806.0102] [INSPIRE].

[60] J. Marsano, N. Saulina and S. Schäfer-Nameki, F-theory compactifications for supersymmetric GUTs, JHEP 08 (2009) 030 [arXiv:0904.3932] [INSPIRE].

[61] J. Marsano, N. Saulina and S. Schäfer-Nameki, Monodromies, fluxes and compact three-generation F-theory GUTs, JHEP 08 (2009) 046 [arXiv:0906.4672] [INSPIRE]. 
[62] R. Blumenhagen, T.W. Grimm, B. Jurke and T. Weigand, Global F-theory GUTs, Nucl. Phys. B 829 (2010) 325 [arXiv:0908.1784] [INSPIRE].

[63] T.W. Grimm, S. Krause and T. Weigand, F-theory GUT vacua on compact Calabi-Yau fourfolds, JHEP 07 (2010) 037 [arXiv: 0912.3524] [INSPIRE].

[64] M. Cvetič, I. Garcia-Etxebarria and J. Halverson, Global F-theory models: instantons and gauge dynamics, JHEP 01 (2011) 073 [arXiv:1003.5337] [INSPIRE].

[65] C.-M. Chen, J. Knapp, M. Kreuzer and C. Mayrhofer, Global SO(10) F-theory GUTs, JHEP 10 (2010) 057 [arXiv: 1005.5735] [INSPIRE].

[66] J. Knapp, M. Kreuzer, C. Mayrhofer and N.-O. Walliser, Toric construction of global F-theory GUTs, JHEP 03 (2011) 138 [arXiv:1101.4908] [INSPIRE].

[67] L. Lin and T. Weigand, Towards the standard model in F-theory, arXiv:1406.6071 [INSPIRE].

[68] D. Klevers, D.K. Mayorga Pena, P.-K. Oehlmann, H. Piragua and J. Reuter, F-theory on all toric hypersurface fibrations and its Higgs branches, arXiv:1408.4808 [INSPIRE].

[69] D.R. Morrison and D.S. Park, F-theory and the Mordell-Weil group of elliptically-fibered Calabi-Yau threefolds, JHEP 10 (2012) 128 [arXiv:1208.2695] [INSPIRE].

[70] M.B. Green, J.H. Schwarz and P.C. West, Anomaly free chiral theories in six-dimensions, Nucl. Phys. B 254 (1985) 327 [INSPIRE].

[71] A. Sagnotti, A note on the Green-Schwarz mechanism in open string theories, Phys. Lett. B 294 (1992) 196 [hep-th/9210127] [INSPIRE].

[72] V. Sadov, Generalized Green-Schwarz mechanism in F-theory, Phys. Lett. B 388 (1996) 45 [hep-th/9606008] [INSPIRE].

[73] J. Erler, Anomaly cancellation in six-dimensions, J. Math. Phys. 35 (1994) 1819 [hep-th/9304104] [INSPIRE].

[74] V. Kumar, D.R. Morrison and W. Taylor, Global aspects of the space of $6 D N=1$ supergravities, JHEP 11 (2010) 118 [arXiv:1008.1062] [INSPIRE].

[75] V. Kumar, D.S. Park and W. Taylor, $6 D$ supergravity without tensor multiplets, JHEP 04 (2011) 080 [arXiv: 1011.0726] [INSPIRE].

[76] S.H. Katz, D.R. Morrison and M.R. Plesser, Enhanced gauge symmetry in type-II string theory, Nucl. Phys. B 477 (1996) 105 [hep-th/9601108] [INSPIRE].

[77] T.W. Grimm and T. Weigand, On Abelian gauge symmetries and proton decay in global F-theory GUTs, Phys. Rev. D 82 (2010) 086009 [arXiv: 1006.0226] [InSPIRE].

[78] D.S. Park and W. Taylor, Constraints on 6D supergravity theories with Abelian gauge symmetry, JHEP 01 (2012) 141 [arXiv:1110.5916] [INSPIRE].

[79] D.S. Park, Anomaly equations and intersection theory, JHEP 01 (2012) 093 [arXiv:1111.2351] [INSPIRE].

[80] M. Cvetič, T.W. Grimm and D. Klevers, Anomaly cancellation and Abelian gauge symmetries in F-theory, JHEP 02 (2013) 101 [arXiv:1210.6034] [INSPIRE].

[81] C. Mayrhofer, E. Palti and T. Weigand, U(1) symmetries in F-theory GUTs with multiple sections, JHEP 03 (2013) 098 [arXiv:1211.6742] [INSPIRE]. 
[82] V. Braun, T.W. Grimm and J. Keitel, New global F-theory GUTs with U(1) symmetries, JHEP 09 (2013) 154 [arXiv:1302.1854] [InSPIRE].

[83] J. Borchmann, C. Mayrhofer, E. Palti and T. Weigand, Elliptic fibrations for $\mathrm{SU}(5) \times \mathrm{U}(1) \times \mathrm{U}(1)$ F-theory vacua, Phys. Rev. D 88 (2013) 046005 [arXiv: 1303.5054] [INSPIRE].

[84] M. Cvetič, D. Klevers and H. Piragua, F-theory compactifications with multiple U(1)-factors: constructing elliptic fibrations with rational sections, JHEP 06 (2013) 067 [arXiv:1303.6970] [INSPIRE].

[85] J. Borchmann, C. Mayrhofer, E. Palti and T. Weigand, SU(5) tops with multiple U(1)s in F-theory, Nucl. Phys. B 882 (2014) 1 [arXiv:1307.2902] [InSPIRE].

[86] M. Cvetič, D. Klevers, H. Piragua and P. Song, Elliptic fibrations with rank three Mordell-Weil group: F-theory with $\mathrm{U}(1) \times \mathrm{U}(1) \times \mathrm{U}(1)$ gauge symmetry, JHEP 03 (2014) 021 [arXiv:1310.0463] [inSPIRE].

[87] M. Cvetič, A. Grassi, D. Klevers and H. Piragua, Chiral four-dimensional F-theory compactifications with $\mathrm{SU}(5)$ and multiple $\mathrm{U}(1)$-factors, JHEP 04 (2014) 010 [arXiv:1306.3987] [INSPIRE].

[88] A.P. Braun, A. Collinucci and R. Valandro, The fate of U(1)'s at strong coupling in F-theory, JHEP 07 (2014) 028 [arXiv: 1402.4054] [INSPIRE].

[89] M.R. Douglas, D.S. Park and C. Schnell, The Cremmer-Scherk mechanism in F-theory compactifications on K3 manifolds, JHEP 05 (2014) 135 [arXiv:1403.1595] [INSPIRE].

[90] D.R. Morrison and W. Taylor, Sections, multisections and U(1) fields in F-theory, arXiv: 1404.1527 [INSPIRE].

[91] C. Schoen, On fiber products of rational elliptic surfaces with section, Math. Z. 197 (1988) 177.

[92] D.R. Morrison, D. Park and W. Taylor, to appear.

[93] E. Witten, On flux quantization in M-theory and the effective action, J. Geom. Phys. 22 (1997) 1 [hep-th/9609122] [INSPIRE].

[94] S. Gukov, C. Vafa and E. Witten, CFT's from Calabi-Yau four folds, Nucl. Phys. B 584 (2000) 69 [Erratum ibid. B 608 (2001) 477] [hep-th/9906070] [INSPIRE].

[95] J. Marsano and S. Schäfer-Nameki, Yukawas, G-flux and spectral covers from resolved Calabi-Yau's, JHEP 11 (2011) 098 [arXiv:1108.1794] [INSPIRE].

[96] T.W. Grimm and H. Hayashi, F-theory fluxes, chirality and Chern-Simons theories, JHEP 03 (2012) 027 [arXiv:1111.1232] [INSPIRE].

[97] J. Halverson, Anomaly nucleation constrains SU(2) gauge theories, Phys. Rev. Lett. 111 (2013) 261601 [arXiv:1310.1091] [InSPIRE].

[98] E. Witten, An SU(2) anomaly, Phys. Lett. B 117 (1982) 324 [InSPIRE].

[99] S. Kachru, R. Kallosh, A.D. Linde and S.P. Trivedi, De Sitter vacua in string theory, Phys. Rev. D 68 (2003) 046005 [hep-th/0301240] [INSPIRE].

[100] V. Balasubramanian, P. Berglund, J.P. Conlon and F. Quevedo, Systematics of moduli stabilisation in Calabi-Yau flux compactifications, JHEP 03 (2005) 007 [hep-th/0502058] [INSPIRE]. 
[101] L. Susskind, The anthropic landscape of string theory, hep-th/0302219 [INSPIRE].

[102] M. Graña, Flux compactifications in string theory: a comprehensive review, Phys. Rept. 423 (2006) 91 [hep-th/0509003] [INSPIRE].

[103] M.R. Douglas and S. Kachru, Flux compactification, Rev. Mod. Phys. 79 (2007) 733 [hep-th/0610102] [INSPIRE].

[104] F. Denef, M.R. Douglas and S. Kachru, Physics of string flux compactifications, Ann. Rev. Nucl. Part. Sci. 57 (2007) 119 [hep-th/0701050] [INSPIRE].

[105] A. Giryavets, S. Kachru and P.K. Tripathy, On the taxonomy of flux vacua, JHEP 08 (2004) 002 [hep-th/0404243] [INSPIRE].

[106] O. DeWolfe, A. Giryavets, S. Kachru and W. Taylor, Enumerating flux vacua with enhanced symmetries, JHEP 02 (2005) 037 [hep-th/0411061] [INSPIRE].

[107] W. Taylor, On the Hodge structure of elliptically fibered Calabi-Yau threefolds, JHEP 08 (2012) 032 [arXiv: 1205.0952] [INSPIRE].

[108] P. Candelas, E. Perevalov and G. Rajesh, Toric geometry and enhanced gauge symmetry of F-theory/heterotic vacua, Nucl. Phys. B 507 (1997) 445 [hep-th/9704097] [INSPIRE].

[109] T. Gherghetta, C.F. Kolda and S.P. Martin, Flat directions in the scalar potential of the supersymmetric standard model, Nucl. Phys. B 468 (1996) 37 [hep-ph/9510370] [INSPIRE].

[110] Y.-H. He, V. Jejjala, C. Matti and B.D. Nelson, Veronese geometry and the electroweak vacuum moduli space, Phys. Lett. B 736 (2014) 20 [arXiv:1402.3312] [INSPIRE].

[111] J.A. Casas, Charge and color breaking, Adv. Ser. Direct. High Energy Phys. 21 (2010) 469 [hep-ph/9707475] [INSPIRE].

[112] S. Abel and T. Falk, Charge and color breaking in the constrained MSSM, Phys. Lett. B 444 (1998) 427 [hep-ph/9810297] [INSPIRE].

[113] T. Cohen and J.G. Wacker, Here be dragons: the unexplored continents of the CMSSM, JHEP 09 (2013) 061 [arXiv: 1305.2914] [INSPIRE].

[114] M. Cvetič, J. Halverson and P. Langacker, Implications of string constraints for exotic matter and $Z^{\prime}$ s beyond the standard model, JHEP 11 (2011) 058 [arXiv:1108.5187] [INSPIRE].

[115] N. Seiberg and W. Taylor, Charge lattices and consistency of 6D supergravity, JHEP 06 (2011) 001 [arXiv: 1103.0019] [INSPIRE]. 